\title{
Photoinduced Structural Phase Transitions in Polyacene
}

\author{
Shoji Yamamoto \\ Department of Physics, Hokkaido University, Sapporo 060-0810, Japan
}

(Dated: 23 March 2011)

\begin{abstract}
There exist two types of structural instability in polyacene: double bonds in a cis pattern and those in a trans pattern. They are isoenergetic but spectroscopically distinct. We demonstrate optical characterization and manipulation of Peierls-distorted polyacene employing both correlated and uncorrelated Hamiltonians. We clarify the phase boundaries of the cis- and trans-distorted isomers, elucidate their optical-conductivity spectra, and then explore their photoresponses. There occurs a photoinduced transformation in the polyacene structure, but it is one-way switching: The trans configuration is well convertible into the cis one, whereas the reverse conversion is much less feasible. Even the weakest light irradiation can cause a transition of uncorrelated electrons, while correlated electrons have a transition threshold against light irradiation.
\end{abstract}

PACS numbers: 78.30.Jw, 78.20.Bh, 78.20.Ci, 71.30.+h

\section{INTRODUCTION}

Since Salem and Longuet-Higgins pointed out that the Peierls instability in polyacene ${ }^{1}$ should be conditional and thus distinct from that in polyacetylene ${ }^{2}$, its ground state has been studied by numerous theoreticians with a variety of numerical tools. Polyacene consists of linearly fused benzene rings and is the next simplest polymer to polyacetylene. Nevertheless, the order of the energetic stability in its structural isomers is still under discussion. The aromatic (AM) configuration of regular lattice closely competes with two types of Peierls-distorted structure: double bonds in a cis pattern and those in a trans pattern, which read as in-phase (IP) and outof-phase (OP) bond order waves (BOWs) in a couple of rows, respectively.

Standard- and extended-Hückel crystalline-orbital calculations at an early stage $e^{\underline{3}-\underline{5}}$ pointed to the OP-BOW phase as the most stable. Band-structure calculations on a modified-neglect-of-diatomic-overlap (MNDO) basis 6.7 followed to find the IP-BOW phase instead to be the most stable. While modern computational approaches such as quantum Monte Carlo $\underline{8}$ and density-matrix renormalization group $\underline{9}$ also supported the IP-BOW phase favored over the OP-BOW one, there exist not a few arguments $\frac{10}{14}$ that IP and OP BOWs are almost isoenergetic. They are indeed degenerate with each other unless we take account of the electron itinerancy beyond the nearest-neighbor hopping 15 and/or the electronic corre-

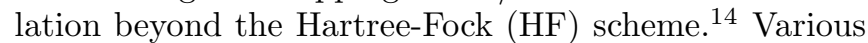
$a b$ initio calculations $16-20$ further show that whether and how polyacene undergoes lattice distortion critically depend on the supposed electronic correlation and the imposed boundary condition. There is thus every reason to believe that the cis- and trans-distorted isomers as well as the regular structure may coexist due to local defects and/or thermal excitations.

Then a query, is there any idea of designing the electronic structure of polyacene other than energetically?
We demonstrate that optics can be the answer. We stimulate a renewed interest in polyacene: optical observation and manipulation of its isomers rather than energetically ranking them.

There are transition-metal-based ladder materials and some of them ${ }^{21-23}$ may also be interesting in the context of varied structural instabilities. Among others are platinum-halide doublechain compounds, where charge-density-wave (CDW) states of the IP and OP types are both realized. $(\mu$-bpym $)\left[\mathrm{Pt}(\right.$ en $) \mathrm{Cl}_{2} \mathrm{Cl}\left(\mathrm{ClO}_{4}\right)_{3} \cdot \mathrm{H}_{2} \mathrm{O} \quad(\mu$-bpym $=$ 2, $2^{\prime}$-bipyrimidine $=\mathrm{C}_{8} \mathrm{H}_{6} \mathrm{~N}_{4}$; en = ethylendiamine $=$ $\left.\mathrm{C}_{2} \mathrm{H}_{8} \mathrm{~N}_{2}\right)^{24}$ and (bpy) $\left[\mathrm{Pt}(\text { dien }) \mathrm{Br}_{2}\right]_{2} \mathrm{Br}_{4} \cdot 2 \mathrm{H}_{2} \mathrm{O}$ (bpy = $4,4^{\prime}$-bipyridyl $=\mathrm{C}_{10} \mathrm{H}_{8} \mathrm{~N}_{2}$; dien $=$ diethylentriamine $=$ $\mathrm{C}_{4} \mathrm{H}_{13} \mathrm{~N}_{3} \stackrel{25}{\underline{2}}$ were fabricated in an attempt to bring into interaction a couple of alternating platinum-halide linear chains via bridging organic ligands, whose platinum ions are of mixed valence and form IP and OP CDWs, respectively $\underline{26,27}$ The intersite electron-phonon coupling brings $\mathrm{BOW}$ varieties into the hydrocarbon ladders, while the intrasite electron-phonon coupling brings CDW varieties into the platinum-halide ladders. Indeed the IPCDW and OP-CDW states also exhibit distinct optical features $\stackrel{28}{2}$ but they are never yet found in a single material. Because they are made in different energy structures, $\stackrel{26}{=}$ they are much less exchangeable with each other whether by light irradiation $\underline{29}$ or by applying pressure ${ }^{30}$. On the other hand, the IP-BOW and OB$\mathrm{BOW}$ states in polyacene are isoenergetic and thus able to coexist. Here is an increasing possibility of optically tuning optical properties.

\section{MODELING}

In order to elucidate the electronic correlation effect on the optical properties, we describe polyacene with and without Coulomb interactions, generally writing the Hamiltonian as 


$$
\begin{aligned}
\mathcal{H}= & -\sum_{l=1}^{2} \sum_{n=1}^{N} \sum_{s= \pm}\left[\left(t_{\|}-\alpha r_{l: 2 n-1}\right) c_{l: 2 n-1, s}^{\dagger} c_{l: 2 n, s}+\left(t_{\|}-\alpha r_{l: 2 n}\right) c_{l: 2 n, s}^{\dagger} c_{l: 2 n+1, s}+\text { H.c. }\right] \\
& -t_{\perp} \sum_{n=1}^{N} \sum_{s= \pm}\left(c_{1: 2 n-1, s}^{\dagger} c_{2: 2 n-1, s}+\text { H.c. }\right)+\frac{K}{2} \sum_{l=1}^{2} \sum_{n=1}^{N}\left(r_{l: 2 n-1}^{2}+r_{l: 2 n}^{2}\right)+\frac{M}{2} \sum_{l=1}^{2} \sum_{n=1}^{N}\left(\dot{u}_{l: 2 n-1}^{2}+\dot{u}_{l: 2 n}^{2}\right) \\
& +U \sum_{l=1}^{2} \sum_{n=1}^{N}\left[\left(n_{l: 2 n-1,+}-\frac{1}{2}\right)\left(n_{l: 2 n-1,-}-\frac{1}{2}\right)+\left(n_{l: 2 n,+}-\frac{1}{2}\right)\left(n_{l: 2 n,-}-\frac{1}{2}\right)\right] \\
& +V_{\|} \sum_{l=1}^{2} \sum_{n=1}^{N} \sum_{s, s^{\prime}= \pm}\left(n_{l: 2 n, s}-\frac{1}{2}\right)\left(n_{l: 2 n-1, s^{\prime}}+n_{l: 2 n+1, s^{\prime}}-1\right)+V_{\perp} \sum_{n=1}^{N} \sum_{s, s^{\prime}= \pm}\left(n_{1: 2 n-1, s}-\frac{1}{2}\right)\left(n_{2: 2 n-1, s^{\prime}}-\frac{1}{2}\right)
\end{aligned}
$$

where $c_{l: j, s}^{\dagger}$ and $c_{l: j, s}\left(c_{l: j, s}^{\dagger} c_{l: j, s} \equiv n_{l: j, s}\right)$ create and annihilate, respectively, a $\pi$ electron of spin $s=\uparrow, \downarrow \equiv \pm$ at site $j$ on chain $l$, while $r_{l: j} \equiv u_{l: j+1}-u_{l: j}$ signifies the bond distortion caused by the $j$ th and $(j+1)$ th carbon atoms on the $l$ th chain. We assign a standard value, $2.4 \mathrm{eV}, \underline{8,9,31,32}$ for the average intrachain hopping integral $t_{\|}$and work at a sufficiently low temperature, $k_{\mathrm{B}} T / t_{\|}=0.01$. If we set the Coulomb interactions all equal to zero, eq. (10) is reduced to the SuSchrieffer-Heeger (SSH)-type Hamiltonian, 33 which is efficient enough to illuminate the structural instability and excitation mechanism of polyacene ${ }^{12,13,34-38}$ In the SSH modeling, the electron-lattice coupling constant $\alpha$ and the $\sigma$-bond elastic constant $K$ are usually set equal to $4.1 \mathrm{eV} / \AA$ and $15.5 \mathrm{eV} / \AA^{2}, 12,13,34$ respectively, together with the carbon-site mass $M$ of $1350 \mathrm{eV} \cdot \mathrm{fs}^{2} / \AA^{2}, \underline{36,39}$ We further set the interchain hopping integral $t_{\perp}$ equal to $0.864 t_{\|}$considering that $\mathrm{C}-\mathrm{C}$ bonds in the rung $(\perp)$ and leg $(\|)$ directions are made in different lengths as $a_{\|}=1.4 \AA<a_{\perp}=1.5 \AA \stackrel{18,20,31,40}{2}$ The full expression (11) is regarded as an extended Peierls-Hubbard (EPH) Hamiltonian,,$\underline{41}$ where we design the Coulomb parameters as $V_{\|(\perp)}=U / \kappa \sqrt{1+0.6117 a_{\|(\perp)}^{2}} \cdot \frac{42}{4} \kappa$ reads as a dielectric parameter and may range from 1 of bare correlation $31,32,43$ to 2 of fully screened correlation ${ }^{32.43}$ Here we consider the intermediate case of $\kappa=1.5$ in an attempt to survey potential ground states and typical photodynamics in linear polybenzenoid hydrocarbons.

We are interested in the generic behavior of polyacene rather than individual features of small oligoacenes. In view of the applicability of the periodic boundary condition and the feasibility of simulating the electron-lattice dynamics on a large scale, we restrict Coulomb interactions to a certain range. We indeed learn from quantum Monte Carlo ${ }^{8}$ and density-matrix renormalizationgroup ${ }^{9}$ calculations that distant Coulomb interactions have little effect on the ground-state properties, including the conditional Peierls instability and the energetics of the structural isomers. We have carried out tentative calculations of both static and dynamic properties for 16, 32, 64, and 128 benzene rings. Optical features such as the absolute energy and relative intensity of every absorption are well converging even at $N=16$. Qualitatively the same dynamics is available as a function of the excitation density for $N \gtrsim 32$. Therefore, we present typical findings at $N=64$ unless otherwise noted.

Figure 1 reveals possible ground states in polyacene. When the electron-lattice and electron-electron interactions are both weak, we find a metallic state of AM configuration. With growing coupling and correlation, the electrons are localized into an antiferromagnetic (AF) Mott insulator (MI) and a bond-alternating Peierls insulator, respectively. A CDW state also appears in the correlated region. Such a close competition between AF $\mathrm{MI}$ and $\mathrm{CDW}$ is due to the moderately valued screening effect. CDW is always favored over AF MI at $\kappa=1, \frac{6}{,}$ whereas vice versa at $\kappa=2 \underline{14}$ Though the Peierls instability in polyacene is conditional, the well-established SSH parameters are known to result in a gapped ground state [cf. Fig. 2(a)]. The two types of Peierls insulator, IP and OP BOWs, are degenerate in energy. Even though Coulomb interactions are switched on, they remain isoenergetic with their Peierls gap being enhanced [cf. Fig. 2(b)]. Keeping the SSH parameters as they are, we turn the Coulomb interactions on. We discuss the correlated dynamics typically at $U=4.8 \mathrm{eV}$ and inquire further whether and how it changes getting toward a boundary to the CDW phase.

\section{SPECTROSCOPIC FEATURES OF THE ISOENERGETIC STRUCTURES}

Considering that a charge-transfer excitation costs energy of $\mathrm{eV}$ order, we evaluate the real part of the optical conductivity as

$$
\sigma(\omega)=\frac{\pi}{\omega} \sum_{i}\left|\left\langle E_{i}|\mathcal{J}| E_{0}\right\rangle\right|^{2} \delta\left(E_{i}-E_{0}-\hbar \omega\right),
$$

where $\left|E_{i}\right\rangle$ denotes the wave vector of the $i$ th-lying state with energy $E_{i}$ and $\mathcal{J}$ signifies the current operator, which is defined in the leg and rung directions, respec- 

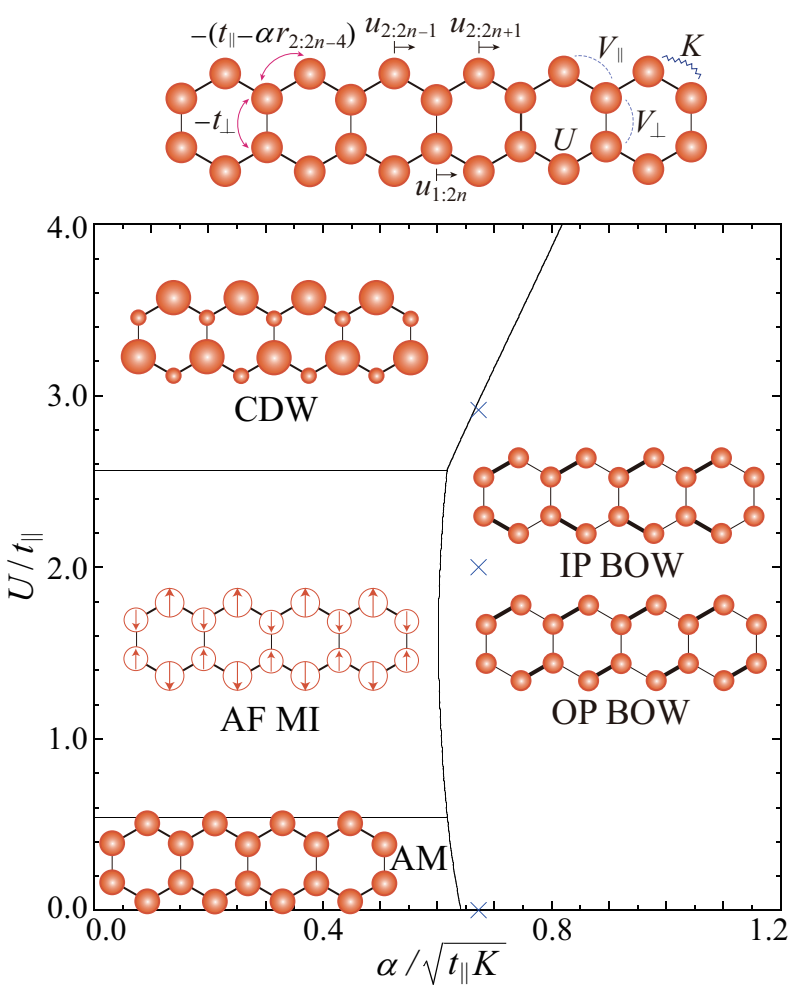

FIG. 1: (Color) A ground-state phase diagram on the $\alpha-U$ plain within the HF approximation. Crosses point to the SSH parameters without any correlation and the EPH parameters in the cases of $U=4.8 \mathrm{eV}$ and $U=7.0 \mathrm{eV}$.

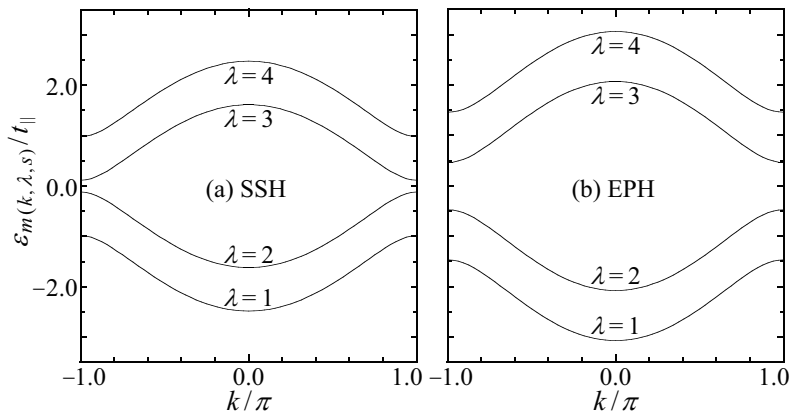

FIG. 2: Dispersion relations of the $\pi$-electron valence $(\lambda=$ $1,2)$ and conduction $(\lambda=3,4)$ bands based on the SSH modeling (a) and the $U=4.8 \mathrm{eV}$ EPH modeling within the HF approximation (b).

tively, as

$$
\begin{aligned}
\mathcal{J}_{\|}= & \frac{\sqrt{3} i e a_{\|}}{2 \hbar} \sum_{l, n, s}\left[\left(t_{\|}-\alpha r_{l: 2 n-1}\right) c_{l: 2 n-1, s}^{\dagger} c_{l: 2 n, s}\right. \\
& \left.+\left(t_{\|}-\alpha r_{l: 2 n}\right) c_{l: 2 n, s}^{\dagger} c_{l: 2 n+1, s}-\text { H.c. }\right], \\
\mathcal{J}_{\perp} & =\frac{i e a_{\|}}{2 \hbar} \sum_{l, n, s}(-1)^{l}\left[\left(t_{\|}-\alpha r_{l: 2 n-1}\right) c_{l: 2 n-1, s}^{\dagger} c_{l: 2 n, s}\right. \\
& \left.-\left(t_{\|}-\alpha r_{l: 2 n}\right) c_{l: 2 n, s}^{\dagger} c_{l: 2 n+1, s}-\text { H.c. }\right] \\
& +\frac{i e a_{\perp}}{\hbar} \sum_{n, s} t_{\perp}\left(c_{1: 2 n-1, s}^{\dagger} c_{2: 2 n-1, s}-\text { H.c. }\right) .
\end{aligned}
$$

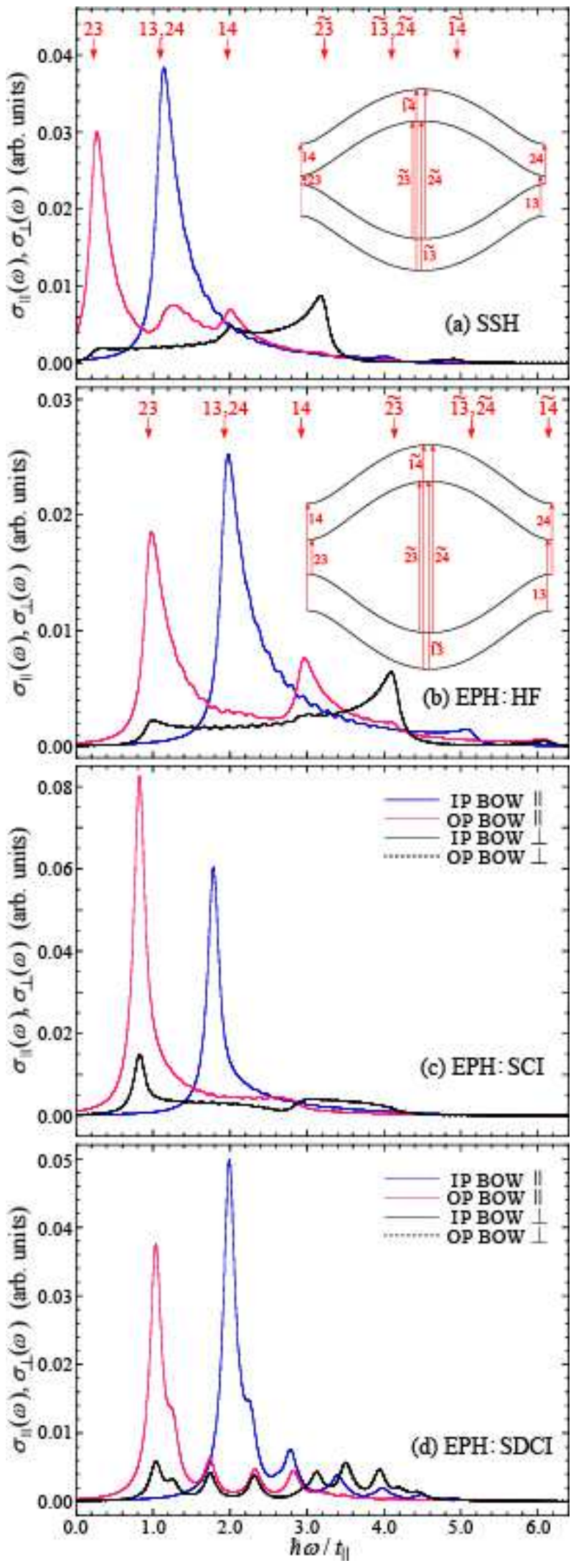

FIG. 3: (Color) Polarized optical conductivity spectra parallel $(\|)$ and perpendicular $(\perp)$ to the long axis for IP and OP BOWs, where every spectral line is Lorentzian broadened by a width of $0.09 t_{\|}$: Exact calculations based on the SSH modeling (a); HF (b), SCI (c), and SDCI (d) calculations based on the $U=4.8 \mathrm{eV} \mathrm{EPH} \mathrm{modeling.}$ 
The state vector is described in terms of Slater determinants and truncated at the order of double excitations:

$$
\begin{aligned}
& \left|E_{i}\right\rangle=f^{(0)}(i)\left|E_{0}\right\rangle_{\mathrm{HF}}+\sum_{m(k, \mu, s)=1}^{4 N} \sum_{m(k, \nu, s)=4 N+1}^{8 N} f^{(1)}(k, \mu, \nu, s ; i) a_{m(k, \nu, s)}^{\dagger} a_{m(k, \mu, s)}\left|E_{0}\right\rangle_{\mathrm{HF}} \\
& +\sum_{m\left(k_{1}, \mu_{1}, s_{1}\right)>m\left(k_{2}, \mu_{2}, s_{2}\right)=1}^{4 N} \sum_{m\left(k_{1}, \nu_{1}, s_{1}\right)>m\left(k_{2}, \nu_{2}, s_{2}\right)=4 N+1}^{8 N} f^{(2)}\left(k_{1}, k_{2}, \mu_{1}, \mu_{2}, \nu_{1}, \nu_{2}, s_{1}, s_{2} ; i\right) \\
& \times a_{m\left(k_{1}, \nu_{1}, s_{1}\right)}^{\dagger} a_{m\left(k_{2}, \nu_{2}, s_{2}\right)}^{\dagger} a_{m\left(k_{1}, \mu_{1}, s_{1}\right)} a_{m\left(k_{2}, \mu_{2}, s_{2}\right)}\left|E_{0}\right\rangle_{\mathrm{HF}},
\end{aligned}
$$

where $\left|E_{0}\right\rangle_{\mathrm{HF}} \equiv \prod_{m=1}^{4 N} a_{m}^{\dagger}|0\rangle$ is the ground-state $\mathrm{HF}$ wave function with $|0\rangle$ being the true electron vacuum and $a_{m}^{\dagger}$ creating an electron in the $m$ th HF orbital with energy $\varepsilon_{m}$. The orbital counter $m(k, \lambda, s)$ is composed of the wave number $(k=2 \pi n / N ; n=1, \cdots, N)$, band label $(\lambda=1, \cdots, 4)$ and spin quantum number $(s= \pm)$. Since an electron excitation is optically allowed without any momentum transfer, $\stackrel{44}{t}$ the periodic boundary condition serves to reduce the number of excited states to be considered.

Excited states of the HF type consist of a single Slater determinant,,$\underset{46}{46}$ where $f^{(0)}(i)=\delta_{0, i}, f^{(1)}(k, \mu, \nu, s ; i)=$ $\delta_{k \mu \nu s, i}$ and $f^{(2)}\left(k_{1}, k_{2}, \mu_{1}, \mu_{2}, \nu_{1}, \nu_{2}, s_{1}, s_{2} ; i\right)=0$, giving $E_{i}={ }_{\mathrm{HF}}\left\langle E_{0}|\mathcal{H}| E_{0}\right\rangle_{\mathrm{HF}}-\varepsilon_{m(k, \mu, s)}+\varepsilon_{m(k, \nu, s)}$. Those of the CI type read as resonating Slater determinants, $\stackrel{47}{\underline{4}}$ where their coefficients are determined so as to diagonalize the original Hamiltonian (11). Within the single-excitation CI (SCI) scheme, $f^{(0)}(i)$ and $f^{(2)}\left(k_{1}, k_{2}, \mu_{1}, \mu_{2}, \nu_{1}, \nu_{2}, s_{1}, s_{2} ; i\right)$ remain unchanged and therefore only excited states are improved. Here is no correction to the ground state, because no single-excitation Slater determinant is obtained by the Hamiltonian operation on the HF ground state. Only configurations of the double-excitation type can directly interact with the HF ground state. Therefore, it pays most to truncate the series expansion at the present order, which is referred to as the single-double-excitation CI (SDCI) scheme, though the cost of SDCI $\left(\sim N^{6}\right)$ is incomparably larger than those of HF $\left(\sim N^{3}\right)$ and SCI $\left(\sim N^{4}\right)$.

Figure 3 shows comparative calculations of the optical conductivity spectra for IP and OP BOWs, where only the SDCI calculation is carried out at $N=16$. For the light polarized perpendicular to the conjugation direction, the two isoenergetic structures still look alike even in the SDCI scheme. The valence band of $\lambda=1$ and the conduction band of $\lambda=3$ are both composed of molecular orbitals symmetric with respect to the reflection about the plane bisecting every rung bond, whereas those of $\lambda=2$ and $\lambda=4$ consist of molecular orbitals antisymmetric to the reflection. Such properties of PM remain unchanged with bond distortion, whether the cis or trans type, while they do not hold in AF MI and CDW. Therefore, interband electronic excitations of the 1-to3 and 2-to- 4 types make no contribution to the rungdirection optical conductivity in both IP and OP BOWs. On the other hand, a long-axis-polarized photon clearly distinguishes between IP and OP BOWs. In the con- jugation direction, 2-to-3 interband excitations, including the highest-occupied-molecular-orbital (HOMO)-tolowest-unoccupied-molecular-orbital (LUMO) transition, are optically forbidden on an IP-BOW background but allowed on an OP-BOW one. OP BOW can thus absorb long-axis-polarized photons of much lower energy than IP BOW can. The zone-center $(k=\pi) 1$-to-3 and 2to-4 interband excitations are most contributive to the IP-BOW spectra in the conjugation direction, whether correlated or uncorrelated, while the OP-BOW spectra in the conjugation direction qualitatively vary with the relevant electronic correlation. Valence-to-conduction-band excitations of any type are optically allowed at the uncorrelated level, but only near HOMO-to-LUMO transitions survive the strong correlation.

IP and OP BOWs may be coexistent in actual oligoacenes. They both exhibit selective absorption of their own for the light polarized parallel to the conjugation direction, especially under the influence of the electronic correlation. Then it is highly interesting whether and how the isoenergetic structural isomers are photoactive. Sufficiently oriented long acenes may have an application to the optical memory.

\section{PHOTOINDUCED PHASE TRANSITIONS BETWEEN THE ISOENERGETIC STRUCTURES}

We simulate time evolution of a charge-transfer excitation solving the Schrödinger equation

$$
i \hbar \dot{\Psi}_{s}(t)=\mathcal{H}_{s}(t) \Psi_{s}(t)
$$

where the spin- $s$ sector of the Hamiltonian, $\mathcal{H}_{s}(t)$, and a complete set of its wave functions, $\Psi_{s}(t)$, are given by square matrices of degree $4 N$. The SSH Hamiltonian is exactly computable, while the EPH Hamiltonian is treated within the HF approximation. In both cases, defining the time-dependent wave functions as

$$
\Psi_{s}(t)=\left[\begin{array}{ccc}
\psi_{1: 1,1, s}(t) & \cdots & \psi_{1: 1,4 N, s}(t) \\
\psi_{2: 1,1, s}(t) & \cdots & \psi_{2: 1,4 N, s}(t) \\
\vdots & & \vdots \\
\psi_{1: 2 N, 1, s}(t) & \cdots & \psi_{1: 2 N, 4 N, s}(t) \\
\psi_{2: 2 N, 1, s}(t) & \cdots & \psi_{2: 2 N, 4 N, s}(t)
\end{array}\right],
$$

eq. (6) is explicitly written as 


$$
\begin{aligned}
& i \hbar \dot{\psi}_{1: 2 n-1, \iota, s}(t)=-\left[t_{\perp}+V_{\perp} p_{2 n-1, s}^{\perp *}(t)\right] \psi_{2: 2 n-1, \iota, s}(t) \\
& \quad-\left[t_{\|}-\alpha r_{1: 2 n-2}(t)+V_{\|} p_{1: 2 n-2, s}^{\|}(t)\right] \psi_{1: 2 n-2, \iota, s}(t)-\left[t_{\|}-\alpha r_{1: 2 n-1}(t)+V_{\|} p_{1: 2 n-1, s}^{\| *}(t)\right] \psi_{1: 2 n, \iota, s}(t) \\
& \quad+\left\{U\left[d_{1: 2 n-1,-s}(t)-\frac{1}{2}\right]+V_{\|}\left[d_{1: 2 n-2}(t)+d_{1: 2 n}(t)-2\right]+V_{\perp}\left[d_{2: 2 n-1}(t)-1\right]\right\} \psi_{1: 2 n-1, \iota, s}(t), \\
& i \hbar \dot{\psi}_{2: 2 n-1, \iota, s}(t)=-\left[t_{\perp}+V_{\perp} p_{2 n-1, s}^{\perp}(t)\right] \psi_{1: 2 n-1, \iota, s}(t) \\
& \quad-\left[t_{\|}-\alpha r_{2: 2 n-2}(t)+V_{\|} p_{2: 2 n-2, s}^{\|}(t)\right] \psi_{1: 2 n-2, \iota, s}(t)-\left[t_{\|}-\alpha r_{2: 2 n-1}(t)+V_{\|} p_{2: 2 n-1, s}^{\| *}(t)\right] \psi_{2: 2 n, \iota, s}(t) \\
& \quad+\left\{U\left[d_{2: 2 n-1,-s}(t)-\frac{1}{2}\right]+V_{\|}\left[d_{2: 2 n-2}(t)+d_{2: 2 n}(t)-2\right]+V_{\perp}\left[d_{1: 2 n-1}(t)-1\right]\right\} \psi_{2: 2 n-1, \iota, s}(t), \\
& i \hbar \dot{\psi}_{1: 2 n, \iota, s}(t)=-\left[t_{\|}-\alpha r_{1: 2 n-1}(t)+V_{\|} p_{1: 2 n-1, s}^{\|}(t)\right] \psi_{1: 2 n-1, \iota, s}(t)-\left[t_{\|}-\alpha r_{1: 2 n}(t)+V_{\|} p_{1: 2 n, s}^{\| *}(t)\right] \psi_{1: 2 n+1, \iota, s}(t) \\
& \quad+\left\{U\left[d_{1: 2 n,-s}(t)-\frac{1}{2}\right]+V_{\|}\left[d_{1: 2 n-1}(t)+d_{1: 2 n+1}(t)-2\right]\right\} \psi_{1: 2 n, \iota, s}(t), \\
& i \hbar \dot{\psi}_{2: 2 n, \iota, s}(t)=-\left[t_{\|}-\alpha r_{2: 2 n-1}(t)+V_{\|} p_{2: 2 n-1, s}^{\|}(t)\right] \psi_{2: 2 n-1, \iota, s}(t)-\left[t_{\|}-\alpha r_{2: 2 n}(t)+V_{\|} p_{2: 2 n, s}^{\| *}(t)\right] \psi_{2: 2 n+1, \iota, s}(t) \\
& \quad+\left\{U\left[d_{2: 2 n,-s}(t)-\frac{1}{2}\right]+V_{\|}\left[d_{2: 2 n-1}(t)+d_{2: 2 n+1}(t)-2\right]\right\} \psi_{2: 2 n, \iota, s}(t),
\end{aligned}
$$

where the electron-density and bond-order parameters are calculated through

$$
\begin{aligned}
& d_{l: j}(t)=\sum_{s} d_{l: j, s}(t)=\sum_{s} \sum_{\iota}^{\prime}\left|\psi_{l: j, \iota, s}(t)\right|^{2} \\
& p_{l: j}^{\|}(t)=\sum_{s} p_{l: j, s}^{\|}(t)=\sum_{s} \sum_{\iota}^{\prime} \psi_{l: j, \iota, s}^{*}(t) \psi_{l: j+1, \iota, s}(t), \\
& p_{j}^{\perp}(t)=\sum_{s} p_{j, s}^{\perp}(t)=\sum_{s} \sum_{\iota}^{\prime} \psi_{1: j, \iota, s}^{*}(t) \psi_{2: j, \iota, s}(t),
\end{aligned}
$$

with $\sum^{\prime}$ denoting a summation over the occupied levels immediately after the photoexcitation.

Discretizing the time variable as $t_{m}=m \Delta t(m=$ $0,1,2, \cdots)$ with an interval much smaller than the opticalphonon time scale, say, $\Delta t=10^{-3} \sqrt{M / K} \simeq 0.01 \mathrm{fs}$, we integrate eq. (6) step by step:

$$
\Psi_{s}\left(t_{m+1}\right)=\exp \left[-\frac{i \mathcal{H}_{s}\left(t_{m}\right)}{\hbar} \Delta t\right] \Psi_{s}\left(t_{m}\right) .
$$

At $t=t_{0}$, the system is photoexcited, that is, some valence electrons are pumped up into a conduction band across the Peierls gap. In response to the photoinduced interband electronic transition, we immediately construct the initial Hamiltonian $\mathcal{H}_{s}\left(t_{0}\right)$ from the modified electronic parameters $d_{l: j}\left(t_{0}\right), p_{l: j}^{\|}\left(t_{0}\right)$, and $p_{j}^{\perp}\left(t_{0}\right)$ leaving the background lattice untouched. Then $\Psi_{s}\left(t_{0}\right)^{\dagger} \mathcal{H}_{s}\left(t_{0}\right) \Psi_{s}\left(t_{0}\right)$ is no more diagonal and that is why the wave vectors begin to fluctuate. Obtaining the instantaneous eigenfunctions and eigenvalues

$$
\Phi_{s}\left(t_{m}\right)=\left[\begin{array}{ccc}
\phi_{1: 1,1, s}\left(t_{m}\right) & \cdots & \phi_{1: 1,4 N, s}\left(t_{m}\right) \\
\phi_{2: 1,1, s}\left(t_{m}\right) & \cdots & \phi_{2: 1,4 N, s}\left(t_{m}\right) \\
\vdots & & \vdots \\
\phi_{1: 2 N, 1, s}\left(t_{m}\right) & \cdots & \phi_{1: 2 N, 4 N, s}\left(t_{m}\right) \\
\phi_{2: 2 N, 1, s}\left(t_{m}\right) & \cdots & \phi_{2: 2 N, 4 N, s}\left(t_{m}\right)
\end{array}\right],
$$

$$
\Phi_{s}^{\dagger}\left(t_{m}\right) \mathcal{H}_{s}\left(t_{m}\right) \Phi_{s}\left(t_{m}\right)=\left[\begin{array}{lll}
\varepsilon_{1, s}\left(t_{m}\right) & & \\
& \ddots & \\
& & \varepsilon_{4 N, s}\left(t_{m}\right)
\end{array}\right],
$$

from $\mathcal{H}_{s}\left(t_{m}\right)$ at every time step, $\stackrel{48}{\stackrel{4}{2}}$ we can express eq. (12) as

$$
\begin{gathered}
\psi_{l^{\prime}: j^{\prime}, \iota^{\prime}, s}\left(t_{m+1}\right)=\sum_{\iota=1}^{4 N} \sum_{l=1}^{2} \sum_{j=1}^{2 N} \exp \left[-\frac{i \varepsilon_{\iota, s}\left(t_{m}\right)}{\hbar} \Delta t\right] \\
\times \phi_{l^{\prime}: j^{\prime}, \iota, s}\left(t_{m}\right) \phi_{l: j, \iota, s}^{*}\left(t_{m}\right) \psi_{l: j, \iota^{\prime}, s}\left(t_{m}\right) .
\end{gathered}
$$

Once the electronic wave functions deviate from the ground-state equilibrium, the lattice begins to fluctuate correspondingly. Its dynamics is governed by Newton's equation of motion,

$$
\begin{gathered}
M \ddot{u}_{l: j}(t)=K\left[u_{l: j-1}(t)-2 u_{l: j}(t)+u_{l: j-1}(t)\right] \\
\quad+2 \alpha \operatorname{Re}\left[p_{l: j}^{\|}(t)-p_{l: j-1}^{\|}(t)\right] \equiv F_{l: j}(t),
\end{gathered}
$$

and solvable stepwise:

$$
\begin{aligned}
& \dot{u}_{l: j}\left(t_{m+1}\right)=\dot{u}_{l: j}\left(t_{m}\right)+\frac{F_{l: j}\left(t_{m}\right)}{M} \Delta t, \\
& u_{l: j}\left(t_{m+1}\right)=u_{l: j}\left(t_{m}\right)+\dot{u}_{l: j}\left(t_{m}\right) \Delta t .
\end{aligned}
$$

The classical treatment of lattice degrees of freedom is successful in investigating both correlated $\underline{49}$ and uncorrelated ${ }^{50}$ electron-lattice dynamics. We avoid setting any "artificial" fluctuation on the lattice initially but start every calculation from a stationary BOW lattice of the IP $(\sigma=1)$ or OP $(\sigma=-1)$ type:

$$
\sigma^{l} r_{l: j}\left(t_{0}\right)=-\sigma^{l} r_{l: j+1}\left(t_{0}\right), \quad \dot{u}_{l: j}\left(t_{0}\right)=0,
$$

in an attempt to simulate every precursor to structural phase transitions quantitatively. 


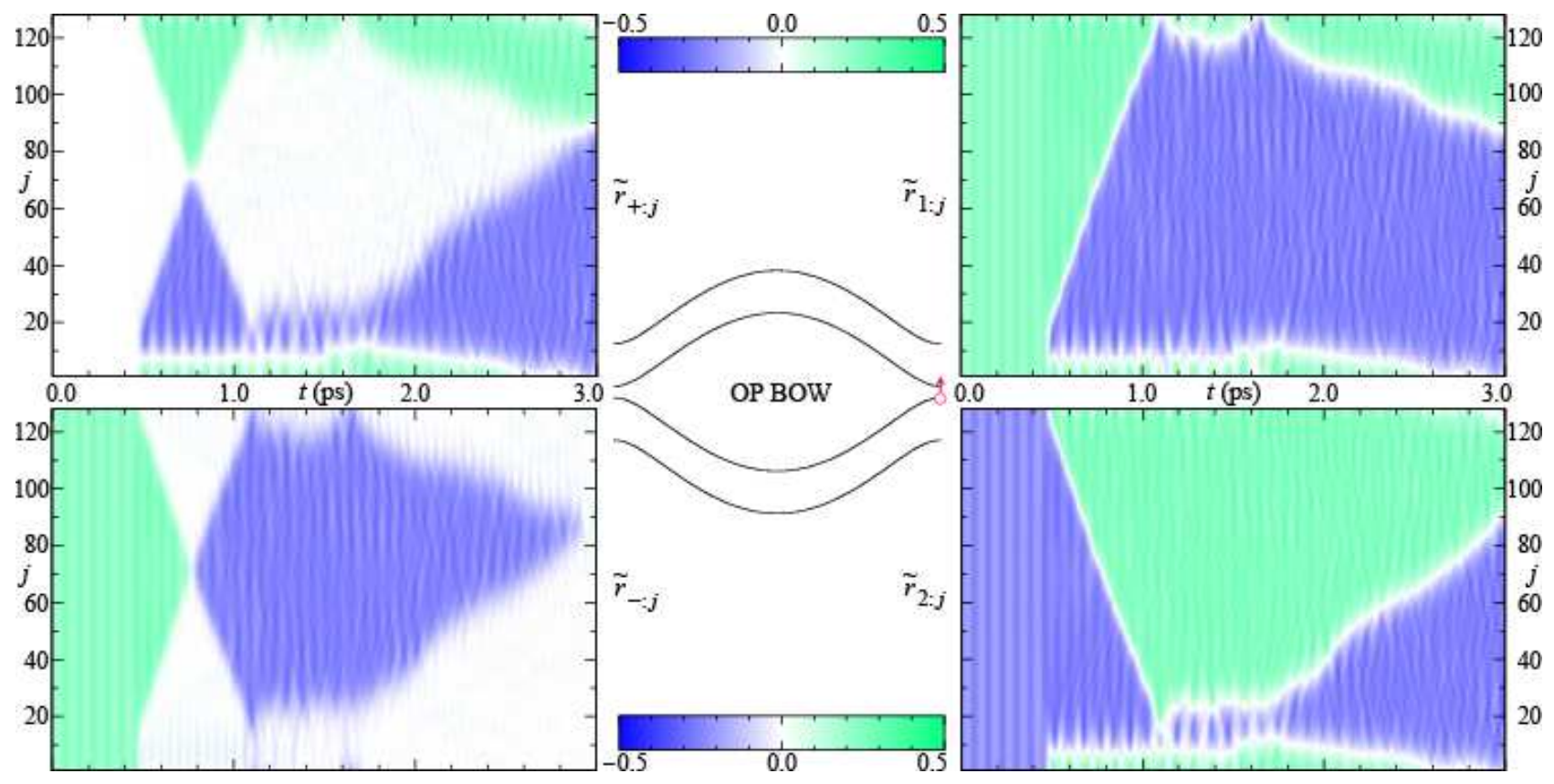

FIG. 4: (Color) SSH-model calculations of the bond variables $\widetilde{r}_{ \pm: j}$ and $\widetilde{r}_{l: j}$ as functions of space $j$ and time $t$ in the case of the HOMO-to-LUMO one-electron excitation on the OP-BOW background.

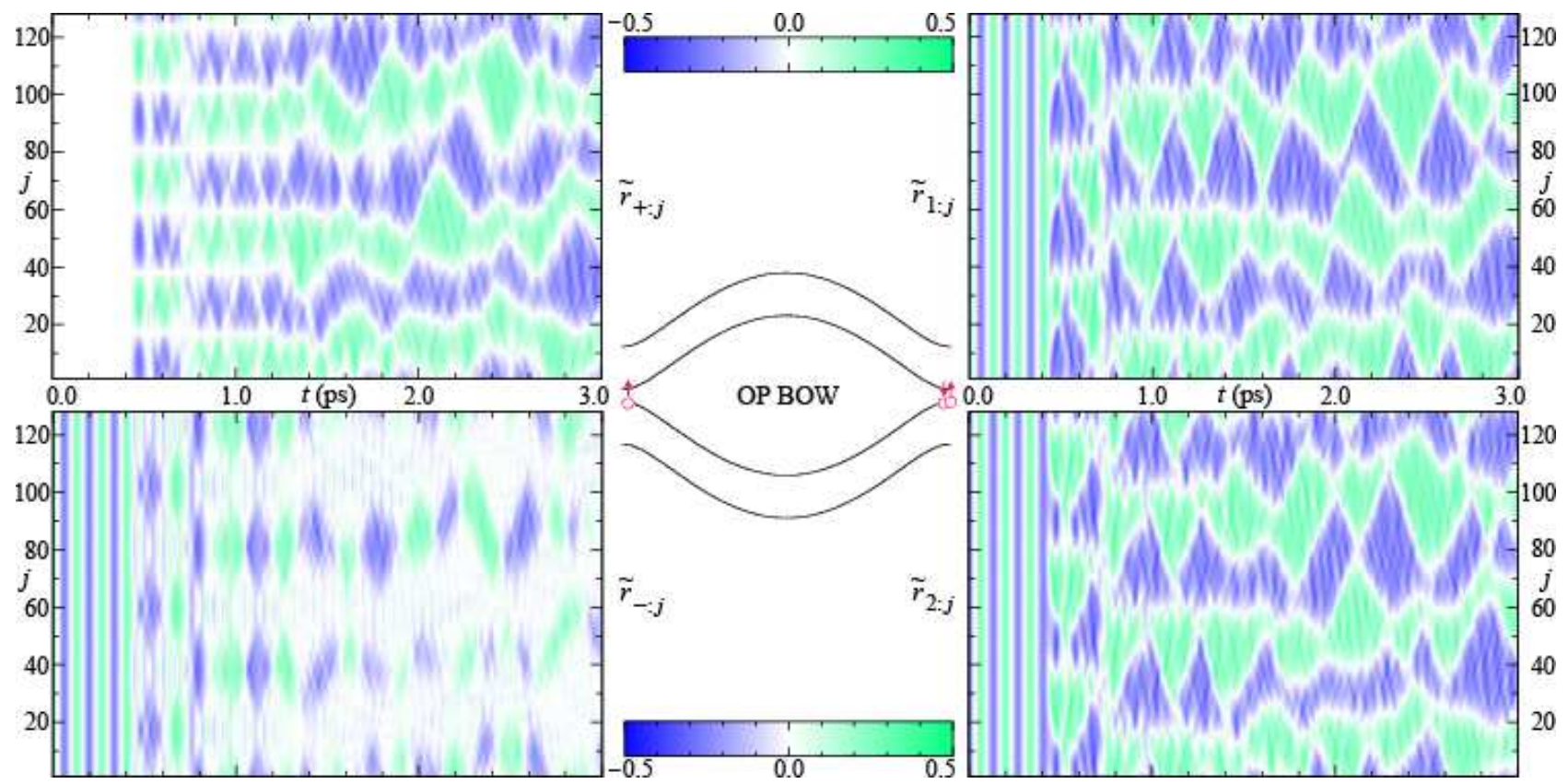

FIG. 5: (Color) The same as Fig. \but in the case of a 2-to-3 interband three-electron excitation at $k \simeq \pi$.

In order to visualize bond arrangements of the IP and OP types, we introduce effective bond variables, $\left(\widetilde{r}_{1: j} \pm \widetilde{r}_{2: j}\right) / 2 \equiv \widetilde{r}_{ \pm: j}$, where the bare bond distortions $r_{l: j}$ are decomposed into their net $\left(\bar{r}_{l: j}\right)$ and alternating $\left(\widetilde{r}_{l: j}\right)$ components as $\bar{r}_{l: j}=\left(2 r_{l: j}+r_{l: j-1}+r_{l: j+1}\right) / 4$ and $\widetilde{r}_{l: j}=\left(2 r_{l: j}-r_{l: j-1}-r_{l: j+1}\right) / 4$. $\widetilde{r}_{l: j}$ remaining constant with varying $j$ read as regular bond alternation on the $l$ th chain, while a change of sign in them denotes a kink (domain wall) occurring. $\widetilde{r}_{+: j}$ and $\widetilde{r}_{-: j}$ serve as detectors of the IP-BOW and OP-BOW configurations, respectively. Now we are ready to observe photoexcitations of polyacene. We consider, unless otherwise noted, a few electrons being excited by around Peierls-gap energy, that is, transitions from the highest one or more occupied to the lowest one or more unoccupied molecular orbitals. The thus-induced electron-lattice dynamics depends on the momentum and spin of the excited electrons but exhibits universal features. 


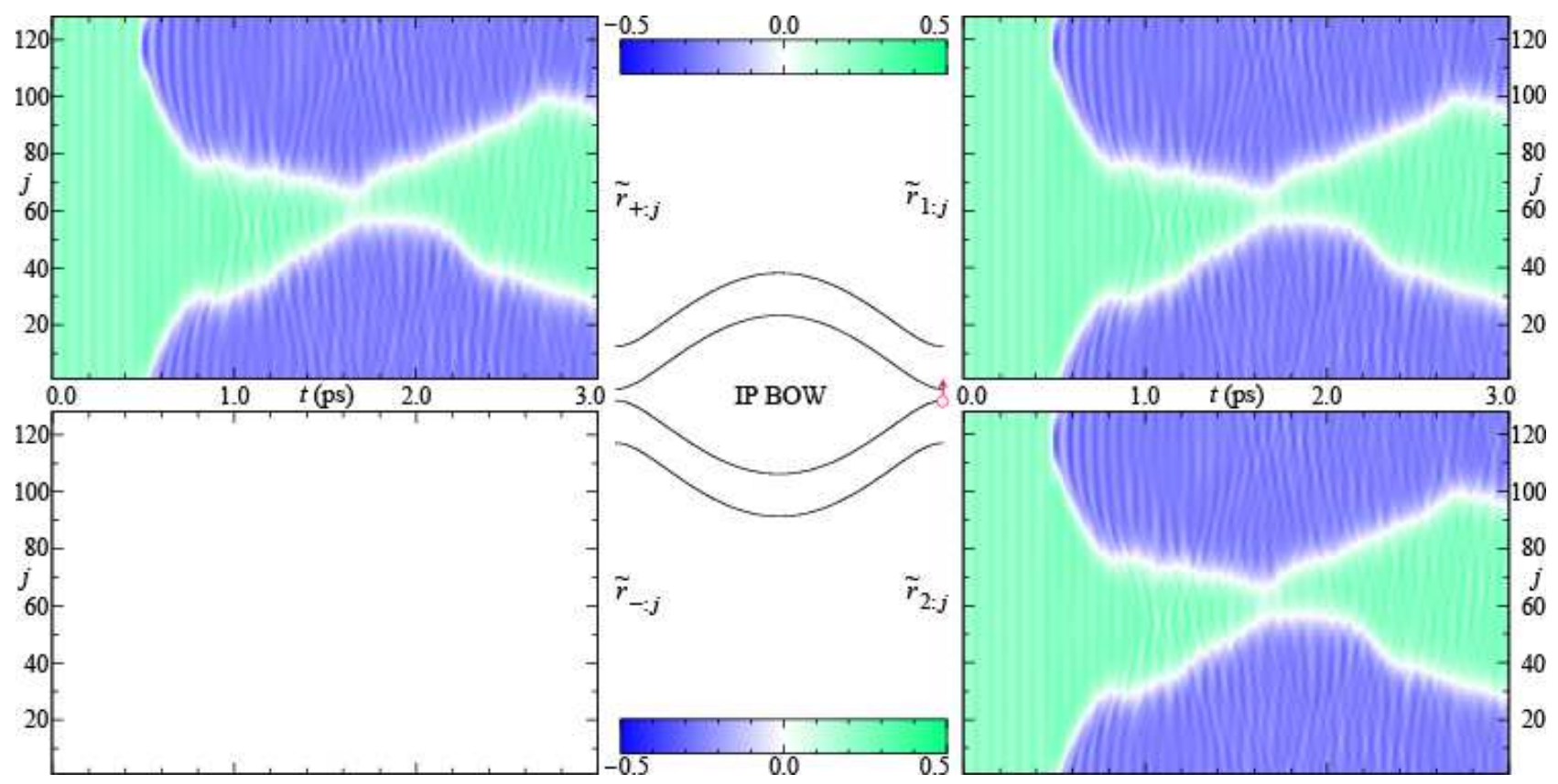

FIG. 6: (Color) The same as Fig. 4 but on the IP-BOW background.
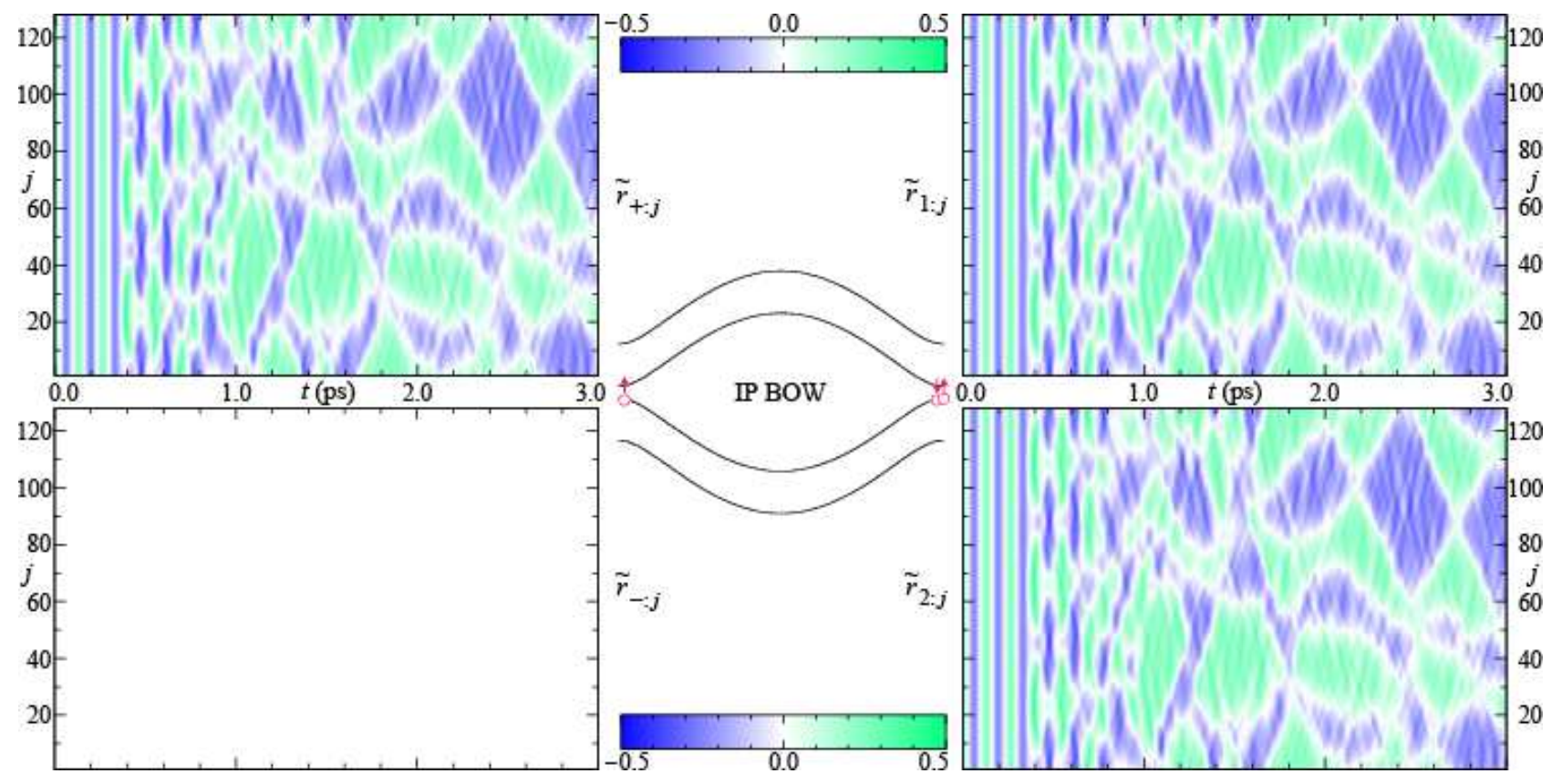

FIG. 7: (Color) The same as Fig. 5 but on the IP-BOW background.

\section{A. Uncorrelated Electron-Lattice Dynamics}

We begin with correlation-free photoresponses. In Fig. 4 we demonstrate the most fundamental HOMO-toLUMO one-electron excitation of the OP-BOW ground state, which is optically allowed in both leg and rung directions. The photon energy is first spent on a background uniform oscillation and then contributes to an OP-to-IP-BOW phase transition. The two bond-ordered chains are disarranged at the same time. The defects in both chains split into a pair of kink and antikink. Then the resultant antidomains grow "repulsively". The outof-phase lattice fluctuations convert the OP-BOW arrangement into the IP-BOW one. The OP-BOW configuration macroscopically recovers within a picosecond but declines again, and finally disappears into the global IP-BOW configuration. Stronger light irradiation drives more electrons to jump over the Peierls gap, induces a precursory global oscillation of larger amplitude and longer period, and then generates multipairs of kink and 


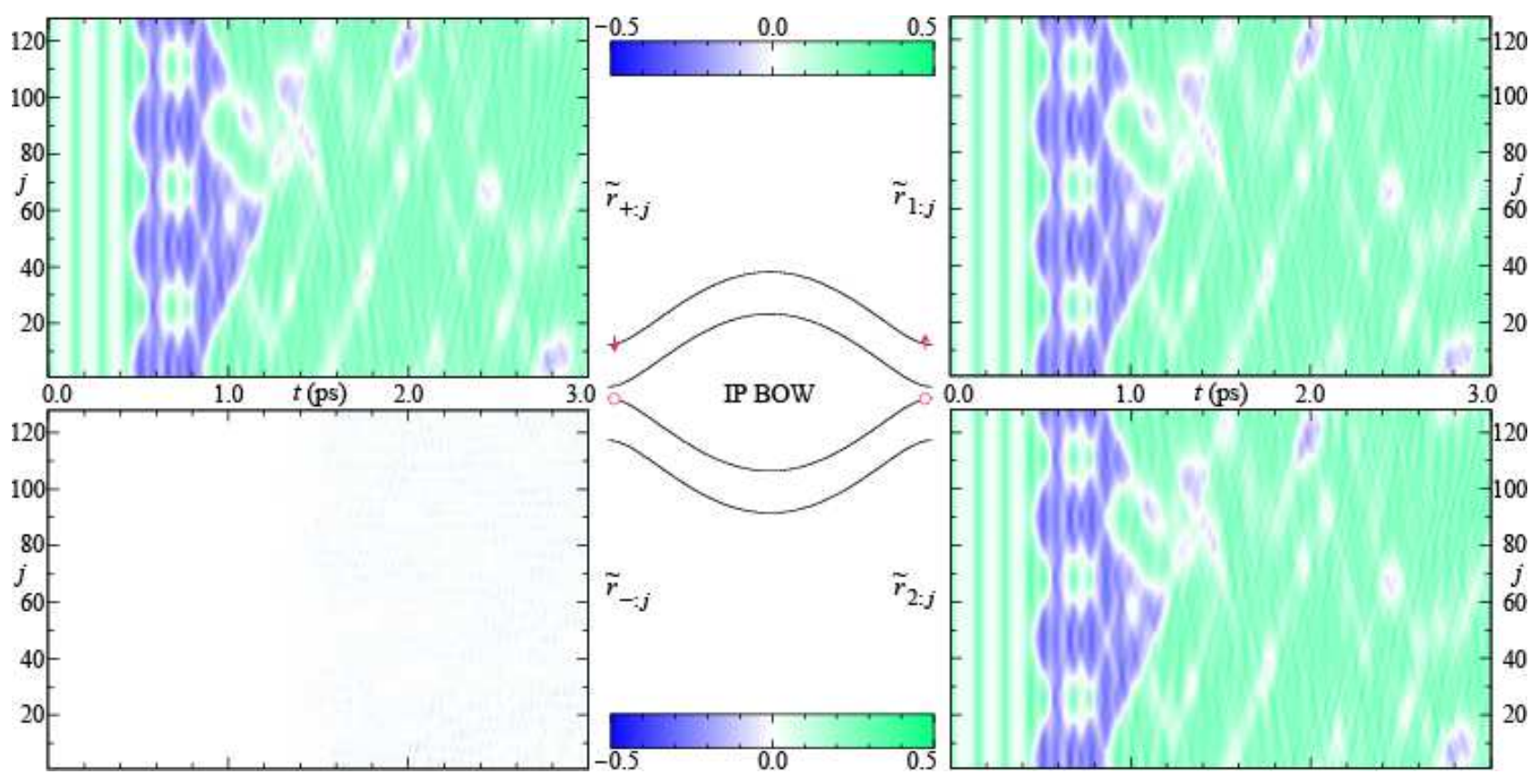

FIG. 8: (Color) The same as Fig. 7 but in the case of a 2-to-4 interband two-electron excitation at $k \simeq \pi$.

antikink against the initial bond arrangement. Figure 5 shows the lattice relaxation from a low-lying threeelectron excited state. Declining and recovering again and again in such a complicated manner, OP BOW disappears into IP BOW within a few picoseconds. Such a swinging motion is observed in a double-chain CDW system without any Coulomb interaction as well ${ }^{51}$ and therefore recognized to be characteristic of uncorrelated electrons.

What happens to IP BOW being photoexcited in the same way? Figure 6 demonstrates the HOMO-to-LUMO one-electron excitation of the IP-BOW ground state and the following lattice relaxation. Indeed the absorbed photon similarly brings a pair of kink and antikink to both chains, but the resultant antidomains now grow "attractively". The kink-antikink breathing motions in the two chains are completely in phase and there is no sign of OP BOW appearing. IP BOW withstands stronger light irradiation. Figure 7 shows that IP BOW with three electrons being excited still keeps itself within a configuration of the IP type. Pumping further electrons up to the lower conduction band, we find no sign of OP-BOW nucleation, even though numbers of antidomains eat away into the initial configuration. Thus excited IP BOW is expected to relax, whether radiatively or nonradiatively, into the initial state via kink-antikink geminate recombinations.

We have so far investigated low-energy excitations of near Peierls gap. OP BOW can be converted to IP BOW by the weakest light irradiation, while IP BOW is light-resistant and survives much stronger photoirradiation. However, there is a possibility of such an inactive response of the IP-BOW state resulting from the light polarization. We should be reminded that a longaxis-polarized photon is not contributive to any inter- band electronic excitation of the 2-to-3 type on an IPBOW background (cf. Fig. 3). Therefore, Figs. 6] and 7 just say that charge-transfer excitations in the rung direction hardly lead to an IP-to-OP bond-structure conversion. We should inquire further into a possibility of phototuning IP BOW by higher-energy excitations. We thus try the 2-to-4 interband one-electron excitation at $k=\pi$, which reads as the optically-allowed lowest-energy charge-transfer excitation in the conjugation direction on an IP-BOW background, but the resultant lattice motion is almost the same as Fig. 6 without any sign of OP-BOW configuration appearing. When we excite further electrons to the upper conduction band (cf. Fig. 8), two chains are no more exactly harmonized with each other. Transient islands of OP-BOW configuration come to appear here and there with increasing number of electrons being excited, but they do not grow up completely. IP BOW looks so persistent and tough against any light irradiation.

\section{B. Correlated Electron-Lattice Dynamics}

Now we switch on Coulomb interactions and discuss correlated-electron-driven lattice dynamics in comparison with the above. When we excite one electron across the Peierls gap on the OP-BOW background in the same manner that we did in Fig. 4 the ground state remains silent without any explicit domain-wall nucleation (Fig. 9). With increasing number of absorbed photons, OP BOW happens to change into IP BOW with three electrons being excited (Fig. 10) but fails to do so even with four electrons being excited (Fig. 11). OP BOW seems to be necessarily converted into IP BOW with more than 


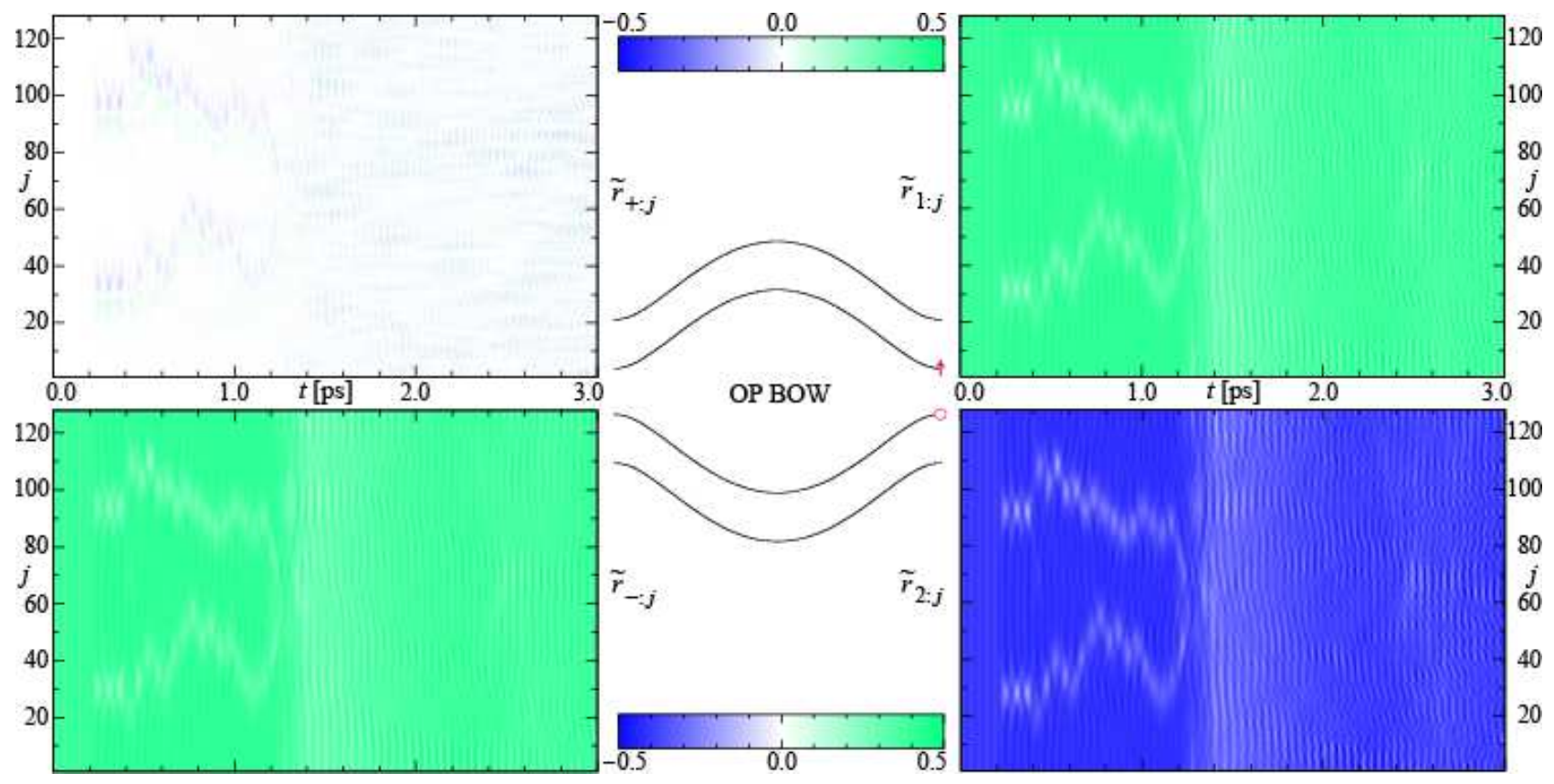

FIG. 9: (Color) $U=4.8 \mathrm{eV}$-EPH-model calculations of the bond variables $\widetilde{r}_{ \pm: j}$ and $\widetilde{r}_{l: j}$ as functions of space $j$ and time $t$ in the case of the HOMO-to-LUMO one-electron excitation on the OP-BOW background.
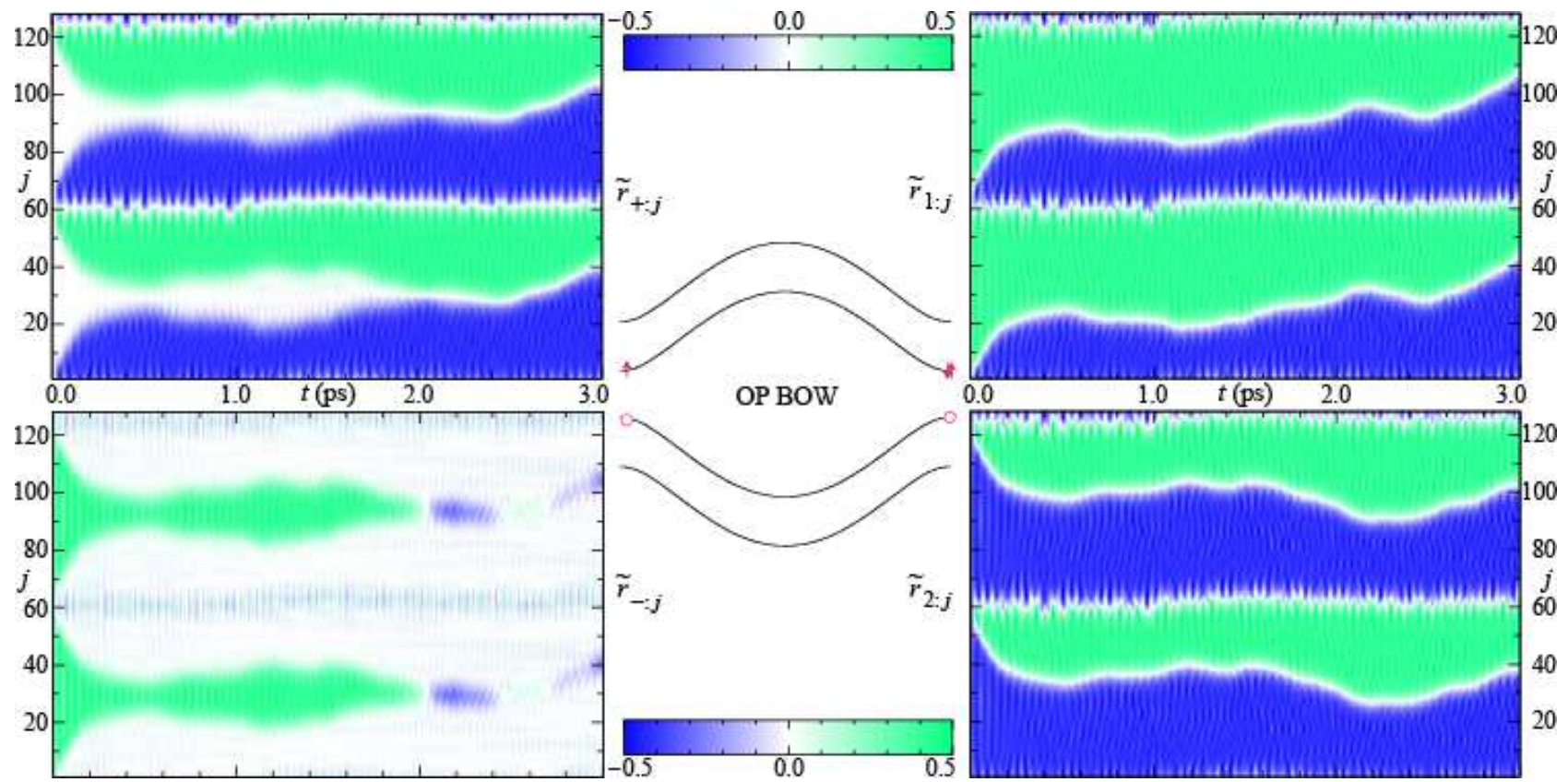

FIG. 10: (Color) The same as Fig. 9 but in the case of a 2-to-3 interband three-electron excitation at $k \simeq \pi$.

four electrons being excited. When electrons are correlated, an OP-to-IP conversion is not feasible until the light irradiation comes up to a certain threshold intensity. Even when an excitation density is given, the correlated dynamics still depends crucially on how electrons are excited. The initial excitation density is determined by the light intensity and a threshold in it, if any, should originate from an energy barrier in the relaxation path. The critical number of excited electrons is not so decisive as it is in a single chain, ${ }^{29}$ because the spatial degrees of freedom in charge-transfer excitations due to the ladder-like lattice structure of polyacene may bypass the relaxation. That is why the threshold excitation density is significantly influenced by the microscopic information about photocarriers such as their momenta and spins.

What about photoexciting IP BOW under the influence of Coulomb interactions? Figure 12 shows the case of three electrons being excited. The observations re- 

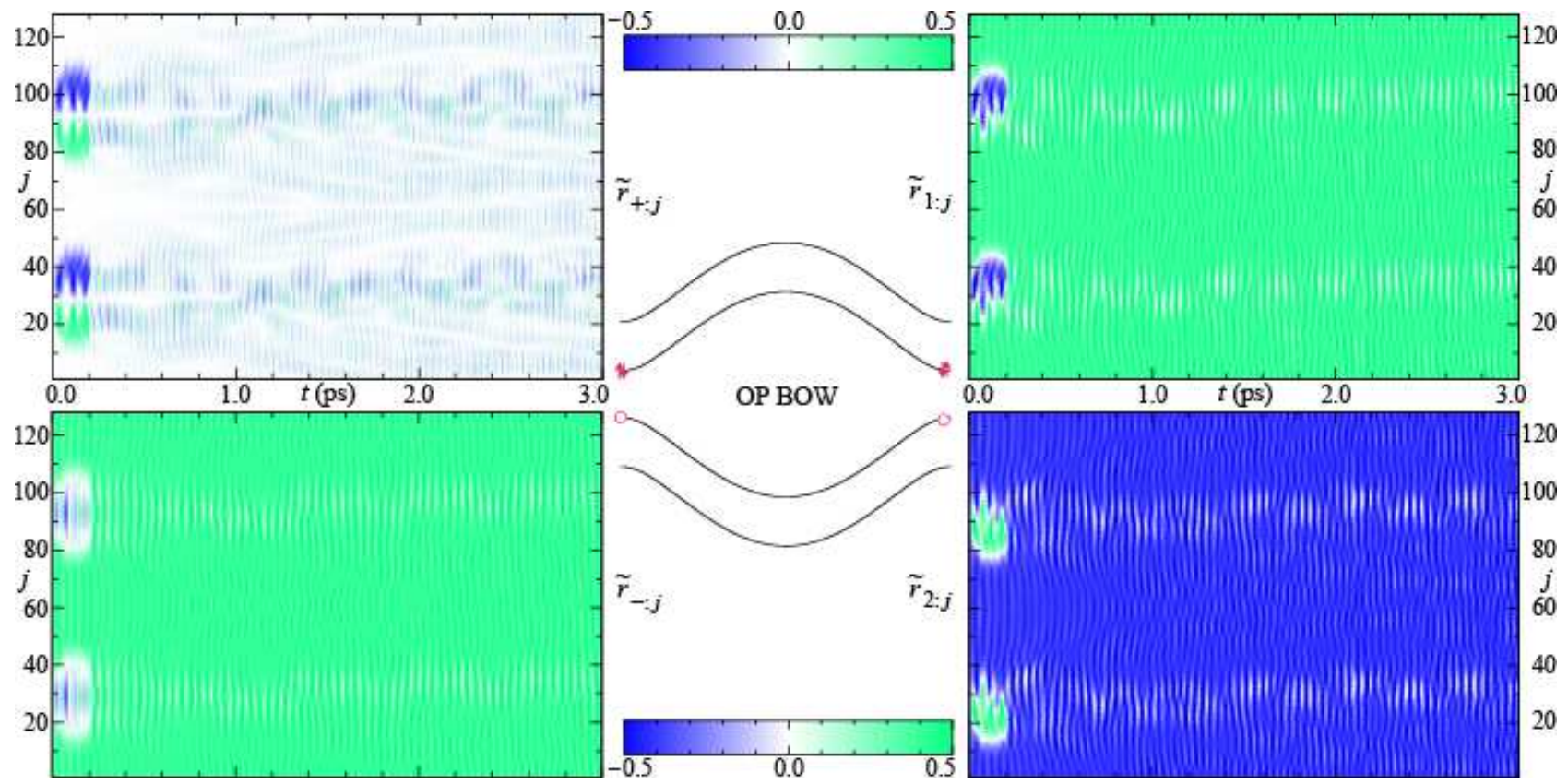

FIG. 11: (Color) The same as Fig. 9 but in the case of a 2 -to-3 interband four-electron excitation at $k \simeq \pi$.
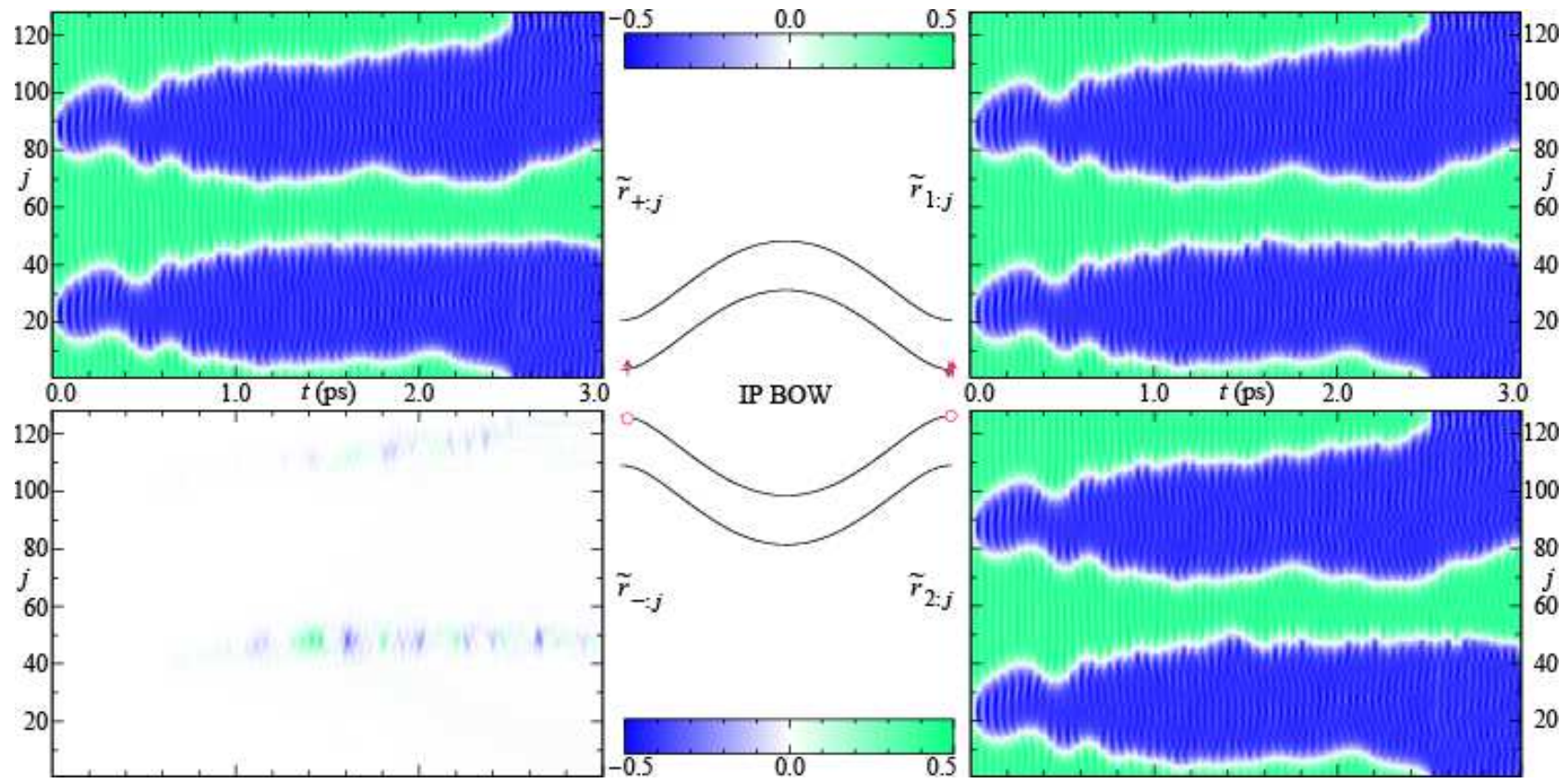

FIG. 12: (Color) The same as Fig. 10 but on the IP-BOW background.

main qualitatively unchanged from the uncorrelated case. It indeed happens that local OP-BOW domains appear with further electrons being excited. However, they hardly aggregate to develop a long-ranged order and decline within several picoseconds. Though the electronic correlation may stimulate local OP-BOW domains to appear, yet a global IP-to-OP-BOW phase transition still looks hard to photoinduce.

Finally we increase the Coulomb interactions so as to get closer to a phase boundary to CDW and pho- toexcite IP and OP BOWs by around Peierls-gap energy. Figure 13 (14) demonstrates the electron-lattice dynamics with four electrons being excited from the OPBOW (IP-BOW) ground state, where we define an effective site variable, $\widetilde{d}_{1: j}-\widetilde{d}_{2: j} \equiv \widetilde{d}_{-: j}$, with $\widetilde{d}_{l: j}=$ $\left(2 d_{l: j}-d_{l: j-1}-d_{l: j+1}\right) / 4$ and plot it as well as $\widetilde{r}_{ \pm: j}$ in order to detect the CDW configuration. In both cases, electrons ultrafast form up into the CDW order and then the BOW lattice arrangement melts down. The liquidized BOW state should fade out if we include the effect of 


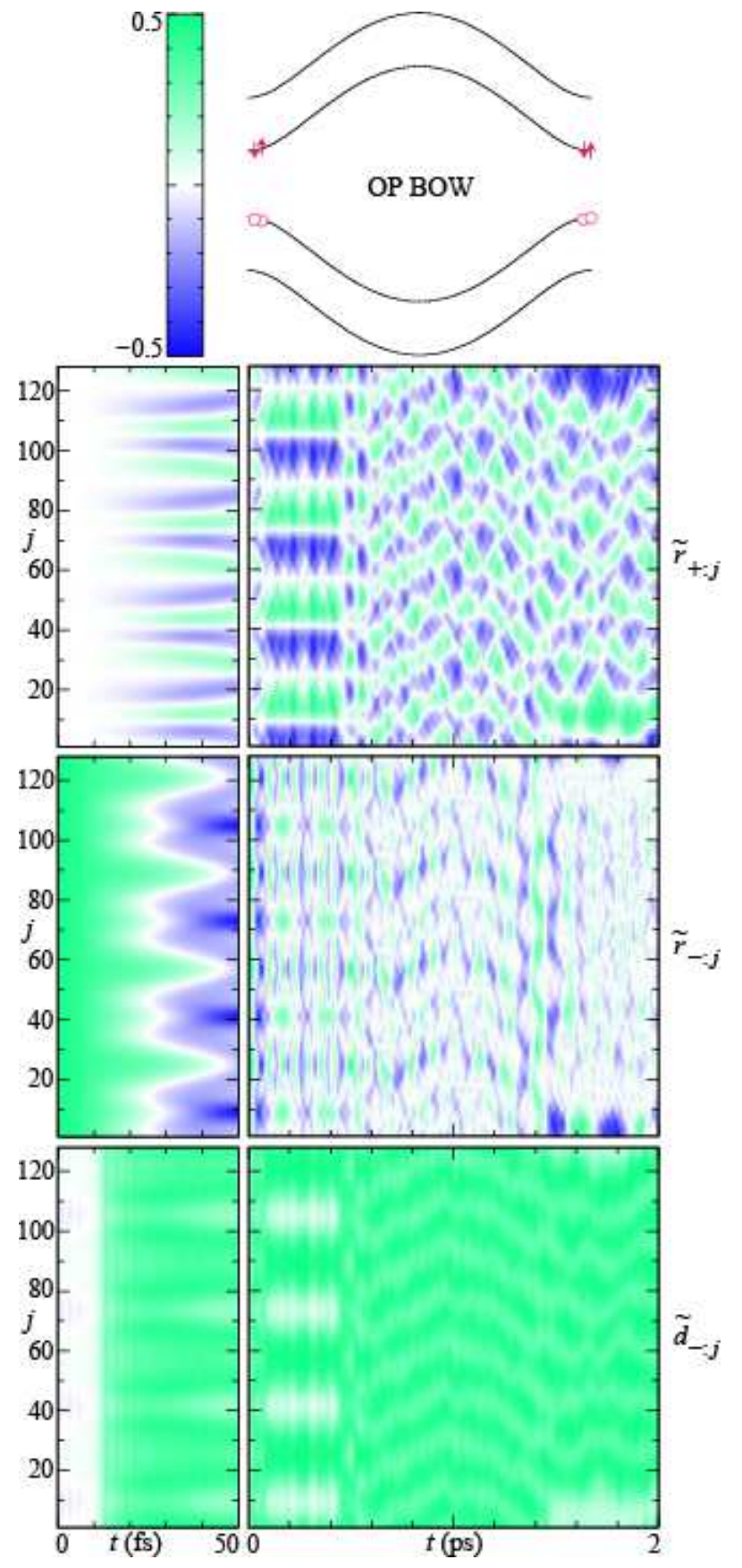

FIG. 13: (Color) $U=7.0 \mathrm{eV}$-EPH-model calculations of the bond variables $\widetilde{r}_{ \pm: j}$ and the site variable $\widetilde{d}_{-: j}$ as functions of space $j$ and time $t$ in the case of a 2-to-3 interband fourelectron excitation at $k \simeq \pi$ on the OP-BOW background. The initial 50 femtoseconds are magnified in an attempt to visualize the ultrafast dynamics in more detail.

extrinsic dissipation into our calculation. A global and surviving CDW order is not attained until the photoexcitation density reaches a certain threshold. The development of the CDW order simply results from closing in upon the phase boundary rather than increasing excitation energy, because such observations are hardly ob-

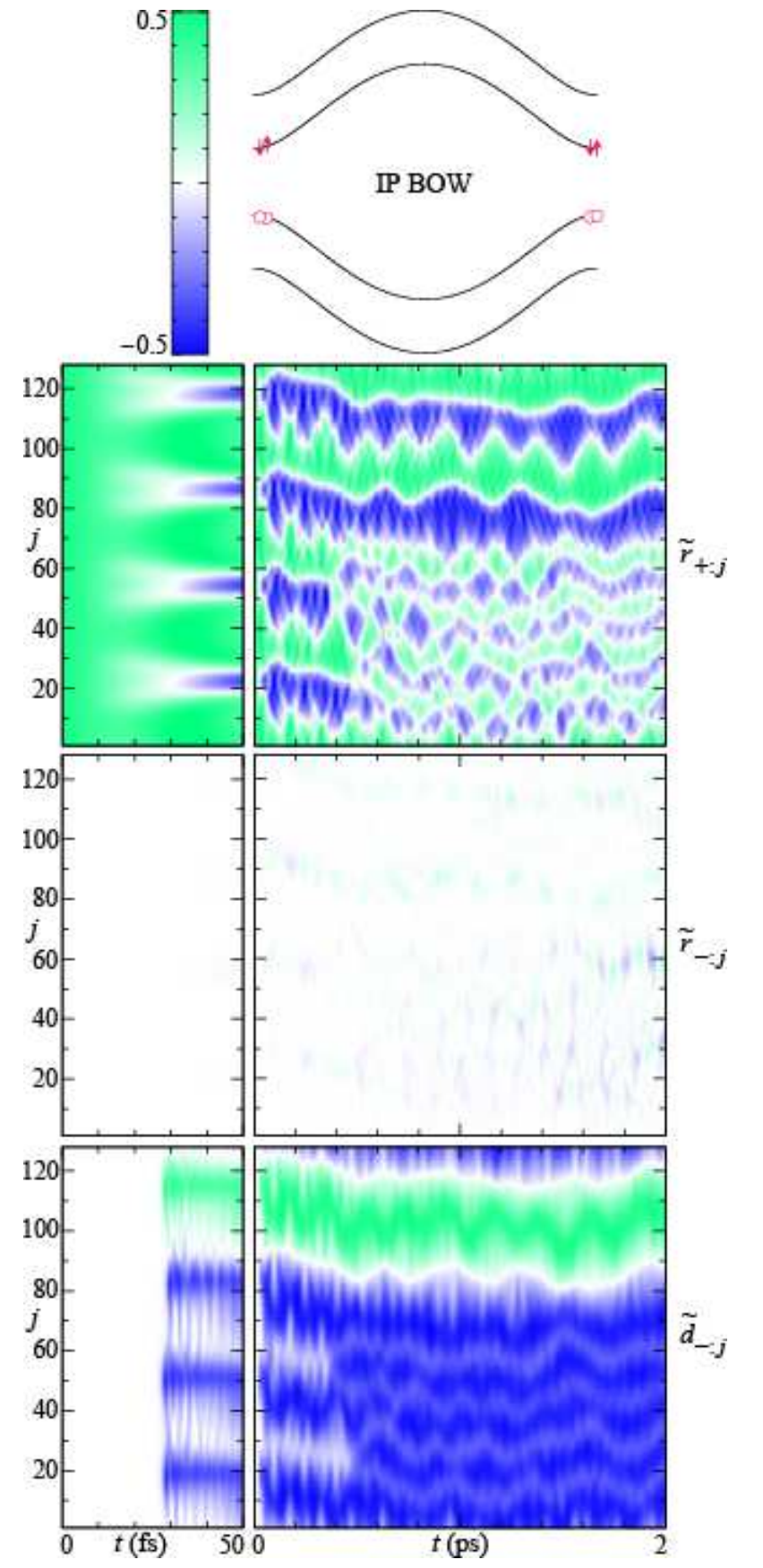

FIG. 14: (Color) The same as Fig. 13 but on the IP-BOW background.

tained from every other previous parametrization. We have indeed tried various photoexcitations, including interband electronic transitions of the 2-to-4 type, in both SSH and $U=4.8 \mathrm{eV}$ EPH models, but such a global $\mathrm{CDW}$ formation is of no occurrence. It is not the BOWto-CDW transition itself but lattice lagging behind electrons in their combined dynamics that interests us here. In this context we may be reminded of a charge-transfer compound, tetrathiafulvalene-p-chloranil, where the electronic valence instability and the dynamic lattice insta- 
bility exhibit different time scales $\frac{52}{2}$ First an ionic domain is photogenerated, whose rising time is so fast as to be less than the experimental resolution, and then the constituent molecules are stimulated to oscillate in a spinPeierls-like manner. Both observations can be characterized as charge transfers preceding lattice oscillations. There is particularity in addition in the present findings. Even in such ultrafast dynamics IP BOW gives an indication of its superiority over OP BOW. OP BOW is once rearranged into IP BOW and then liquidized, while IP BOW directly breaks down without explicit nucleation of OP BOW.

\section{SUMMARY AND DISCUSSION}

We have optically characterized and manipulated the two isoenergetic structures of polyacene with bond arrangements of the IP and OP types. An OP-to-IP-BOW phase transition can be induced by the weakest light irradiation unless electrons are correlated. Coulomb interactions function as energy barriers in the relaxation path and then the transition is conditioned on a good number of electrons being excited. On the other hand, an IP-to-OP-BOW phase transition is much less caused by photoexcitations, whether electrons are correlated or not. IP and OP BOWs may coexist in polyacene. If the Peierls-distorted polyacene is irradiated with light of around Peierls-gap energy, the mixed ground state is possibly unified into a monostructure of the IP-BOW type. The photoinduced predominant state can be annealed back into the bimodal configuration. Figure 3 shows that IP and OP BOWs selectively absorb ultraviolet and blue-green rays, respectively, provided $t_{\|}=2.4 \mathrm{eV}$. Polyacene thus reveals itself as a photochromic polymer ${ }^{53}$ with novel structural bistability.

Photoinduced charge-transfer excitations potentially develop into kink-antikink bound pairs. Their breathing motions can cause a global phase change. Figure 11, demonstrating geminate recombinations of domain walls in the early stage, implies that correlated electrons encounter energy barriers on the way of photogenerated excitons being self-trapped and further dissociated into distant kinks and antikinks. A correlation-induced energy barrier on the relaxation path to far distant domain walls is observed in platinum-halide chain compounds as well, $46,48,54,55$ which are well known to be photoactive Peierls insulators. Since a valley precedes the barrier, Coulomb interactions stimulate the Frank-Condon state to relax and accelerate its localization. $\stackrel{48}{ }$ We are thus convinced of the ultrafast quasifemtosecond self-localization of photogenerated charge-transfer excitons in the correlated dynamics (Figs. 10 14) and its slowing down in the uncorrelated dynamics (Figs. 48).

Coulomb interactions further serve quick and certain switching. Comparing Figs. [5] and [10, we learn that the electronic correlation suppresses the macroscopic oscillation of going back and forth between IP and OP
BOWs. In order to illuminate such a contrast between correlated and uncorrelated electrons in more detail, we try to quantitatively evaluate the weights of IP-BOW and OP-BOW configurations. Their time-dependent and spatially-varying amplitudes may both be normalized in comparison with the initial uniform BOW, because coexistent stable IP-BOW and OP-BOW solutions have exactly the same amplitude. In the case of photoexciting OP BOW, the normalized momentary strengths of IP-BOW and OP-BOW configurations can be defined as $P_{ \pm}(t) \equiv \sum_{j=1}^{N} \widetilde{r}_{ \pm: j}^{2}(t) / \sum_{j=1}^{N} \widetilde{r}_{-: j}^{2}(0)$. We calculate $P_{ \pm}(t)$ with and without Coulomb interactions and plot them in Fig. 15. Besides the precursory global oscillation without any OP-to-IP-BOW conversion yet, the initial OP-BOW state composed of uncorrelated electrons repeats rise and fall on a macroscopic scale for a picosecond or less. The uncorrelated OP-BOW state is so persistent in its configuration. On the other hand, the initial OP-BOW state of correlated electrons is much more quickly and smoothly replaced by the photoinduced IP-BOW phase once the excitation density reaches the threshold. The promotion of OP-to-IP-BOW conversion is also recognizable as a correlation effect. With a few electrons being excited, the converted fraction amounts to four fifths in the correlated case but stays less than two fifths in the uncorrelated case. Correlated electrons possess an adiabatic potential energy surface full of ups and downs. Once they climb over the energy barriers and find a stable configuration, they are unlikely to swing back to the initial state.

The macroscopic oscillation of uncorrelated electrons in their photoconversion to the IP-BOW state and the correlation-driven globalization of the IP-BOW domain are both understandable by observing kink excitations in more detail. The phase transition originates in generation of kink and antikink in pairs. Within the SSH modeling, neither spin nor charge accompanies any kink as a result of resonating nonlinear excitations, $\stackrel{48,56}{,}$ where neutral and charged kinks are isoenergetic. With increasing Coulomb interactions, their degeneracy is lifted and those with excess spin (charge) density in their centers are relatively stabilized (destabilized). In the EPH calculations some of the kink excitations indeed bear net spins and it is their intrachain interactions that develop and hold the induced IP-BOW arrangement against the OP-BOW background. In a successful OP-to-IP transition [cf. Figs. [10, 15 $\left(\mathrm{c}^{\prime}\right)$, and [15 $\left(\mathrm{d}^{\prime}\right)$ ], we find two or more kinks conveying net spin densities of the same sign in each chain, whereas in a frustrated one [cf. Figs. 9], 15 $\left(a^{\prime}\right)$, and [15 $\left.\left(b^{\prime}\right)\right]$, we observe bound pairs of kink and antikink with excess charge, rather than spin, densities disappear in the early stage. The repulsive same-spincarrying kinks may be the key to a global phase change. On the other hand, SSH kinks without any excess density of spin and charge look hardly correlated and can therefore get closer to each other (cf. Fig. 4). They are more and more created with increasing number of photons being absorbed, but their geminate recombination is of frequent occurrence. That is why the converted fraction $P_{+}$ 

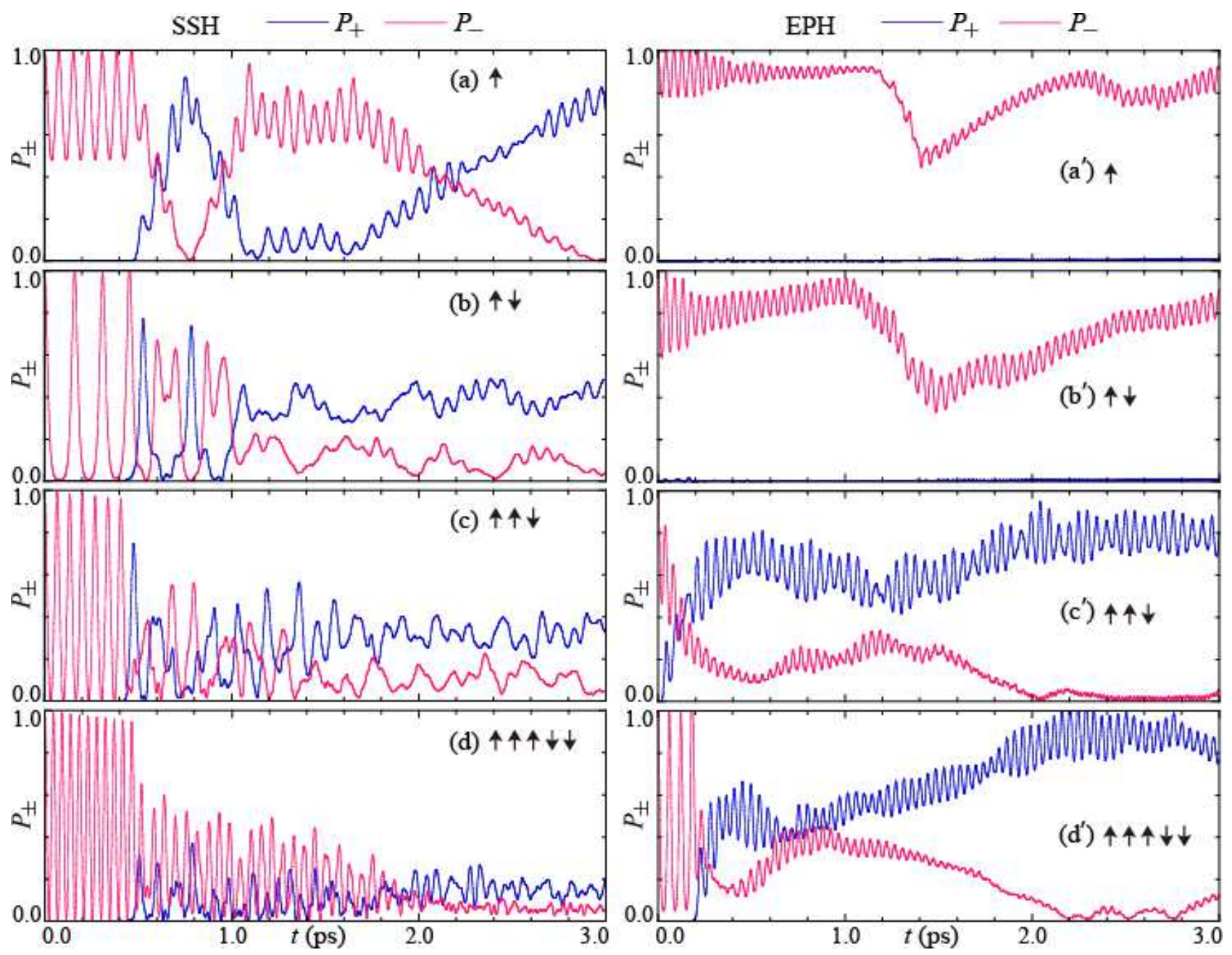

FIG. 15: (Color) Probabilities of finding the IP-BOW and OP-BOW configurations, $P_{ \pm}$, as functions of time $t$ after photoexcitation: SSH-model $[(\mathrm{a})$ to $(\mathrm{d})]$ and $U=4.8 \mathrm{eV}$-EPH-model $\left[\left(\mathrm{a}^{\prime}\right)\right.$ to $\left.\left(\mathrm{d}^{\prime}\right)\right]$ calculations in the cases of 2 -to-3 interband one-electron $\left[(\mathrm{a})\right.$ and $\left.\left(\mathrm{a}^{\prime}\right)\right]$, two-electron $\left[(\mathrm{b})\right.$ and $\left.\left(\mathrm{b}^{\prime}\right)\right]$, three-electron $\left[(\mathrm{c})\right.$ and $\left.\left(\mathrm{c}^{\prime}\right)\right]$ and five-electron $\left[(\mathrm{d})\right.$ and $\left.\left(\mathrm{d}^{\prime}\right)\right]$ excitations at $k \simeq \pi$ on the OP-BOW background. (a) and (c) correspond to Figs. 4 and 5 respectively, while $\left(\mathrm{a}^{\prime}\right)$ and $\left(\mathrm{c}^{\prime}\right)$ to Figs. 9 and 10 , respectively.

oscillates on a macroscopic scale in the SSH modeling [cf. Figs. 15(a)-15(c) in particular]. The reduction of both IP-BOW and OP-BOW strengths with increasing excitation density [Figs. 15(a)-15(d)] is also attributable to the free kinks and thus-unlimited domain-wall nucleation.

A variety of photoinduced phase transitions have so far been observed and analyzed indeed. However, most of them assume the initial condition to be close to the phase boundary and such a situation often depends in quite a critical manner on some structural parameters which are hardly tractable. Oligoacenes possess inherent bistability of topological origin and their Peierlsdistorted structural isomers are strictly and stably isoenergetic. Even under uniaxial pressure, for instance, applied in an attempt to tune their intrinsic properties such as $t_{\perp} / t_{\|}$and $V_{\perp} / V_{\|}$, the strict bistability holds and en- ables us to systematically investigate whether and how energetically degenerate isomers are photoconverted into each other. Polyacene derivatives and analogues such as polyphenanthrene $e^{\frac{4}{-}}$ and polyacenacene $\mathrm{e}^{57}$ will make possible further comparative studies. We are looking forward to more and more interest in this potential organic polymer.

\section{Acknowledgments}

The author is grateful to J. Ohara for useful comments. This work was supported by the Suhara Memorial Foundation and the Ministry of Education, Culture, Sports, Science, and Technology of Japan.
${ }^{1}$ L. Salem and H. C. Longuet-Higgins: Proc. Roy. Soc. A, 255 (1960) 435.
${ }^{2}$ H. C. Longuet-Higgins and L. Salem: Proc. Roy. Soc. A, 
251 (1959) 172.

3 M.-H. Whangbo, R. Hoffmann, and R. B. Woodward: Proc. Roy. Soc. A, 366 (1979) 23.

${ }^{4}$ K. Tanaka, K. Ohzeki, S. Nankai, and T. Yamabe: J. Phys. Chem. Solids 44 (1983) 1069.

5 A. L. S. da Rosa and C. P. de Melo: Phys. Rev. B 38 (1988) 5430.

6 M. Kertesz, Y. S. Lee, and J. J. P. Stewart: Int. J. Mod. Chem. 35 (1989) 305.

7 J. Chandrasekhar and P. K. Das: J. Phys. Chem. 96 (1992) 679.

8 B. Srinivasan and S. Ramasesha: Phys. Rev. B 57 (1998) 8927.

9 C. Raghu, Y. Anusooya Pati and S. Ramasesha: Phys. Rev. B 65 (2002) 155204.

${ }^{10}$ I. Božović: Phys. Rev. B 32 (1985) 8136.

11 M. A. Garcia-Bach, A. Peñaranda, and D. J. Klein: Phys. Rev. B 45 (1992) 10891.

12 M. K. Sabra: Phys. Rev. B 53 (1996) 1269.

13 Z. J. Li, H. Q. Lin, and K. L. Yao: Z. Phys. B 104 (1997) 77.

14 S. Yamamoto: Phys. Rev. B 78 (2008) 235205.

15 R. S. Pfeiffer, G. Yoder, and A.-B. Chen: Phys. Rev. B 54 (1996) 1735.

16 J. Cioslowski: J. Chem. Phys. 98 (1993) 473.

17 K. N. Houk, P.S. Lee, and M. Nendel: J. Org. Chem. 66 (2001) 5517.

18 M. Bendikov, H. M. Duong, K. Starkey, K. N. Houk, E. A. Carter, and F. Wudl: J. Am. Chem. Soc. 126 (2004) 7416.

19 M. C. dos Santos: Phys. Rev. B 74 (2006) 045426.

20 J. Hachmann, J. J. Dorando, M. Avilés, and G. K.-L. Chan: J. Chem. Phys. 127 (2007) 134309.

21 J. Riera and D. Poilblanc: Phys. Rev. B 59 (1999) 2667.

${ }^{22}$ M. Aichhorn, M. Hohenadler, E. Ya. Sherman, J. Spitaler, C. Ambrosch-Draxl, and H. G. Evertz: Phys. Rev. B 69 (2004) 245108.

23 K. Funase and S. Yamamoto: J. Phys. Soc. Jpn. 75 (2006) 044717.

24 D. Kawakami, M. Yamashita, S. Matsunaga, S. Takaishi, T. Kajiwara, H. Miyasaka, K. Sugiura, H. Matsuzaki, and H. Okamoto: Angew. Chem. 118 (2006) 7372.

25 A. Kobayashi and H. Kitagawa: J. Am. Chem. Soc. 128 (2006) 12066.

26 S. Yamamoto and J. Ohara: Phys. Rev. B 76 (2007) 235116 .

27 K. Iwano and Y. Shimoi: J. Phys. Soc. Jpn. 76 (2007) 063708.

28 S. Yamamoto and J. Ohara: J. Mater. Sci.: Mater. Elec- tron. 20 (2009) S367.

29 K. Yonemitsu and N. Miyashita: Phys. Rev. B 68 (2003) 075113.

30 S. Yamamoto: Phys. Rev. B 64 (2001) 140102(R).

31 C. Raghu, Y. Anusooya, and S. Ramasesha: Phys. Rev. B 66 (2002) 035116.

32 P. Sony and A. Shukla: Phys. Rev. B 75 (2007) 155208.

${ }^{33}$ W. P. Su, J. R. Schrieffer, and A. J. Heeger: Phys. Rev. Lett. 42 (1979) 1698.

${ }^{34}$ Y. J. Wu, H. Zhao, Z. An, and C. Q. Wu: J. Phys.: Condens. Matter 14 (2002) L341.

35 Z. An and C. Q. Wu: Int. J. Mod. Phys. B 17 (2003) 2023.

${ }^{36}$ Z. An and C. Q. Wu: Eur. Phys. J. B 42 (2004) 467.

37 H. Zhao, Z. An, and C. Q. Wu: Eur. Phys. J. B 43 (2005) 53.

38 Z. J. Li, H. B. Xu, and K. L. Yao: Mod. Phys. Lett. B 11 (1997) 477.

39 S. Yamamoto and J. Ohara: Physica B 405 (2010) S365.

${ }^{40}$ K. B. Wiberg: J. Org. Chem. 62 (1997) 5720.

41 A. Takahashi, S. Yamamoto, and H. Fukutome: J. Phys. Soc. Jpn. 61 (1992) 199.

42 K. Ohno: Theor. Chim. Acta 2 (1964) 219.

43 A. Shukla, H. Ghosh, and S. Mazumdar: Phys. Rev. B 67 (2003) 245203.

44 P. Huai, H. Zheng, and K. Nasu: J. Phys. Soc. Jpn. 69 (2000) 1788.

45 S. M. Hasanuzzaman, K. Iwano, and K. Nasu: J. Phys. Soc. Jpn. 68 (1999) 1376.

46 J. Ohara and S. Yamamoto: Phys. Rev. B 73 (2006) 045122.

47 A. Shukla: Phys. Rev. B 65 (2002) 125204.

48 S. Yamamoto and J. Ohara: J. Phys.: Condens. Matter 20 (2008) 415215.

49 N. Miyashita, M. Kuwabara, and K. Yonemitsu: J. Phys. Soc. Jpn. 72 (2003) 2282.

50 K. Iwano: Phys. Rev. B 65 (2001) 024302.

51 K. Iwano: Phys. Rev. B 66 (2002) 060302(R).

52 S. Iwai, Y. Ishige, S. Tanaka, Y. Okimoto, Y. Tokura, and H. Okamoto: Phys. Rev. Lett. 96 (2006) 057403.

53 N. Hosaka, H. Tachibana, N. Shiga, M. Matsumoto, and Y. Tokura: Phys. Rev. Lett. 82 (1999) 1672.

54 A. Mishima and K. Nasu: Phys. Rev. B 39 (1989) 5758.

55 K. Iwano: J. Phys. Soc. Jpn. 66 (1997) 1088.

56 Y. Hirano and Y. Ono: J. Phys. Soc. Jpn. 69 (2000) 2131.

57 T. Yamabe, K. Tanaka, K. Ohzeki, and S. Yata: Solid State Commun. 44 (1982) 823. 